\title{
Small deviations for fractional stable processes
}

\author{
Mikhail Lifshits ${ }^{\mathrm{a}, \mathrm{b}, 1}$, Thomas Simon ${ }^{\mathrm{c}, *}$ \\ ${ }^{\text {a }}$ Faculty of Mathematics and Mechanics, St.Petersburg State University, 198504 Stary Peterhof, Bibliotechnaya pl., 2, Russia \\ b Université Lille-I, France \\ c Équipe d'analyse et probabilités, université d'Évry-Val d'Essonne, boulevard François Mitterrand, 91025 Évry Cedex, France
}

Received 26 May 2003; received in revised form 10 May 2004; accepted 1 June 2004

Available online 17 September 2004

\section{Abstract}

Let $\left\{R_{t}, 0 \leqslant t \leqslant 1\right\}$ be a symmetric $\alpha$-stable Riemann-Liouville process with Hurst parameter $H>0$. Consider a translation invariant, $\beta$-self-similar, and $p$-pseudo-additive functional semi-norm $\|\cdot\|$. We show that if $H>\beta+1 / p$ and $\gamma=(H-\beta-$ $1 / p)^{-1}$, then

$$
\lim _{\varepsilon \downarrow 0} \varepsilon^{\gamma} \log \mathbb{P}[\|R\| \leqslant \varepsilon]=-\mathcal{K} \in[-\infty, 0),
$$

with $\mathcal{K}$ finite in the Gaussian case $\alpha=2$. If $\alpha<2$, we prove that $\mathcal{K}$ is finite when $R$ is continuous and $H>\beta+1 / p+1 / \alpha$. We also show that under the above assumptions,

$$
\lim _{\varepsilon \downarrow 0} \varepsilon^{\gamma} \log \mathbb{P}[\|X\| \leqslant \varepsilon]=-\mathcal{K} \in(-\infty, 0),
$$

where $X$ is the linear $\alpha$-stable fractional motion with Hurst parameter $H \in(0,1)$ (if $\alpha=2$, then $X$ is the classical fractional Brownian motion). These general results cover many cases previously studied in the literature, and also prove the existence of new small deviation constants, both in Gaussian and non-Gaussian frameworks.

(C) 2004 Elsevier SAS. All rights reserved.

\section{Résumé}

Soit $\left\{R_{t}, 0 \leqslant t \leqslant 1\right\}$ un processus de Riemann-Liouville $\alpha$-stable symétrique avec paramètre de Hurst $H>0$. Considérons une semi-norme fonctionnelle $\|\cdot\|$ invariante par translation, $\beta$-autosimilaire et $p$-pseudo-additive. Nous montrons que si $H>\beta+1 / p$ et $\gamma=(H-\beta-1 / p)^{-1}$ alors

$$
\lim _{\varepsilon \downarrow 0} \varepsilon^{\gamma} \log \mathbb{P}[\|R\| \leqslant \varepsilon]=-\mathcal{K} \in[-\infty, 0),
$$

\footnotetext{
* Corresponding author.

E-mail addresses: lifts@mail.rcom.ru (M.A. Lifshits), tsimon@univ-evry.fr (T. Simon).

1 Research partially supported by grants RFBR 02-01-00265, 04-01-04000 and NSh-2258.2003.1.
} 
avec $\mathcal{K}$ finie dans le cas gaussien $\alpha=2$. Lorsque $\alpha<2$, nous montrons que $\mathcal{K}$ est finie quand $R$ est continu et $H>\beta+1 / p+$ $1 / \alpha$. Nous montrons aussi que sous ces hypothèses

$$
\lim _{\varepsilon \downarrow 0} \varepsilon^{\gamma} \log \mathbb{P}[\|X\| \leqslant \varepsilon]=-\mathcal{K} \in(-\infty, 0),
$$

où $X$ est le mouvement fractionnaire linéaire $\alpha$-stable avec paramètre de Hurst $H \in(0,1)$ (lorsque $\alpha=2, X$ est le mouvement brownien fractionnaire usuel). Ces résultats généraux recouvrent de nombreux cas précédemment étudiés dans la littérature et prouvent l'existence de nouvelles constantes de petites déviations, aussi bien dans le cadre gaussien que non gaussien.

(c) 2004 Elsevier SAS. All rights reserved.

MSC: 60E07; 60G15; 60G17; 60G18

Keywords: Fractional Brownian motion; Gaussian process; Linear fractional stable motion; Riemann-Liouville process; Small ball constants; Small ball probabilities; Small deviations; Stable process; Wavelets

\section{Introduction}

Let $X$ be a random process whose sample paths belong to some functional normed space $(\mathcal{F},\|\cdot\|)$. The investigation of the small deviations (or small ball probabilities) of $X$ deals with the asymptotics of

$$
\mathbb{P}[\|X\| \leqslant \varepsilon]
$$

when $\varepsilon \downarrow 0$, and has proved to be a difficult problem with increasing number of applications in Probability, Analysis, Complexity... etc. We refer to the recent surveys [26,27] for a detailed account on this subject.

In the literature, this problem is usually studied for a particular class of processes and under a particular norm. It remains a great challenge to find some principle describing small deviations for general classes of processes and norms, rather than investigate the problem case by case.

The unique successful attempt in this direction was made by W. Stolz [41,42], who obtained estimates

$$
-\infty<\liminf _{\varepsilon \downarrow 0} \varepsilon^{\gamma} \log \mathbb{P}[\|X\| \leqslant \varepsilon] \leqslant \limsup _{\varepsilon \downarrow 0} \varepsilon^{\gamma} \log \mathbb{P}[\|X\| \leqslant \varepsilon]<0,
$$

where $\left\{X_{t}, 0 \leqslant t \leqslant 1\right\}$ is a Brownian motion (or more generally a continuous Gaussian process with covariance function similar to that of fractional Brownian motion), and $\gamma$ a finite positive parameter depending on the behavior of the (semi-)norm $\|\cdot\|$ on linear combinations of Schauder functions. A lot of classical semi-norms fell into Stolz's scope: $L_{p}$-norms, Hölder and Sobolev semi-norms, Besov norms... etc., and his estimates provided a general point of view on many previously studied situations (see the references quoted in $[41,42]$ ).

The next important issue is the existence of the limit in (1), and this is the matter of the present paper. Our main result says that if $\|\cdot\|$ is a translation invariant, $\beta$-self-similar and $p$-superadditive functional semi-norm - see Definition 1 for more details about these notions, and $\left\{R_{t}, 0 \leqslant t \leqslant 1\right\}$ is a symmetric $\alpha$-stable Riemann-Liouville process with Hurst parameter $H>\beta+1 / p$ ( $R$ can be viewed as a fractionally integrated symmetric $\alpha$-stable Lévy process, see Section 2 for a precise definition), then

$$
\lim _{\varepsilon \downarrow 0} \varepsilon^{\gamma} \log \mathbb{P}[\|R\| \leqslant \varepsilon]=-\mathcal{K} \in[-\infty, 0)
$$

with the rate $\gamma=(H-\beta-1 / p)^{-1}$. From the technical point of view, the main ingredient of the proof is a stochastic superadditive inequality which is based upon the extrapolation-homogeneity of $R$, and is then easy to combine with an exponential Tauberian theorem and the standard subadditivity arguments.

Our framework has two secondary, but non-negligible advantages with respect to [42]. Non-Gaussian stable processes are included, as well as smooth Gaussian ones like $m$-times integrated Brownian motion. 
A special effort is needed to prove that the constant $\mathcal{K}$ in (2) is finite. In order to get the suitable lower bound on the small deviation probabilities, we use roughly the same method as in [41,42], except that we decompose $R$ along Daubechies' wavelet bases, since Schauder system is not smooth enough when $H$ becomes too large.

In the last part of this paper, we extend the above results to a class of self-similar processes with long range dependence, the so-called unilateral linear fractional stable motions. These processes can be viewed as a possible generalization of fractional Brownian motion with an underlying stable noise. Thanks to a nice argument essentially due to $\mathrm{Li}$ and Linde [23], the problem is reduced to a study of the Schauder decomposition of the associated long memory process.

We conclude this article with a brief survey of concrete results. It seems that our theorems contain everything that is known about the existence of finite small deviation constants for continuous fractional processes under translation-invariant semi-norms. Last but not least, some constants appearing in our results seem to be new, both in Gaussian and non-Gaussian situations.

Still, a major drawback of the above wavelet methods is that they exclude discontinuous processes. As a rule, proving lower bound probabilities for processes with jumps requires completely different discretization techniques $[32,44]$, and we have no idea how to adapt our approach to this case.

\section{Preliminaries}

\subsection{Parametrization: $(\beta, p)$-semi-norms}

Let $\mathcal{I}$ be the set of all closed bounded intervals of $\mathbb{R}$. Consider $\mathcal{F}$ a linear space of functions from $\mathbb{R}$ to $\mathbb{R}$ and, for each $I \in \mathcal{I}$, let $\mathcal{F}_{I}$ be a linear space of functions from $I$ to $\mathbb{R}$ such that $f_{I} \in \mathcal{F}_{I}$ for every $f \in \mathcal{F}$, where $f_{I}$ stands for the restriction of $f$ to $I$.

We define a semi-norm $\|\cdot\|$ on $\mathcal{F}$ as a family $\left\{\|\cdot\|_{I}, I \in \mathcal{I}\right\}$ of functionals mapping $\mathcal{F}_{I}$ to $\mathbb{R}^{+}$such that $\|\lambda f\|_{I}=|\lambda|\|f\|_{I}$ and $\|f+g\|_{I} \leqslant\|f\|_{I}+\|g\|_{I}$ for every $\lambda \in \mathbb{R}, f, g \in \mathcal{F}_{I}$. We will use the notation $\|f\|_{I}=\left\|f_{I}\right\|_{I}$ for every $f \in \mathcal{F}, I \in \mathcal{I}$. In the remainder of this paper we will assume that $\|\cdot\|$ satisfies the following assumptions, which are verified by all the classical semi-norms:

(A) $\|\cdot\|_{I} \leqslant\|\cdot\|_{J}$ for every $I, J \in \mathcal{I}$ such that $I \subset J$. (Contractivity)

(B) $\|f\|_{I-c}=\|f(\cdot-c)\|_{I}$ for every $f \in \mathcal{F}, I \in \mathcal{I}$ and $c \in \mathbb{R}$. (Translation-invariance)

Definition 1. Let $\beta \in \mathbb{R}, p \in(0,+\infty]$ and $\|\cdot\|$ be a contractive and translation-invariant semi-norm on $\mathcal{F}$. We say that $\|\cdot\|$ is an upper $(\beta, p)$-semi-norm if it satisfies the following properties:

(C) $\|f(c \cdot)\|_{I / c}=c^{\beta}\|f\|_{I}$ for every $f \in \mathcal{F}, I \in \mathcal{I}$ and $c>0$. ( $\beta$-self-similarity)

(D) For every $a_{0}<\cdots<a_{n} \in \mathbb{R}$ and $f \in \mathcal{F}$

$$
\begin{cases}\|f\|_{\left[a_{0}, a_{n}\right]} \geqslant\left(\|f\|_{\left[a_{0}, a_{1}\right]}^{p}+\cdots+\|f\|_{\left[a_{n-1}, a_{n}\right]}^{p}\right)^{1 / p} & \text { if } p<+\infty,\end{cases}
$$

In the following we will denote by $\mathbf{U}(\beta, p)$ the set of upper $(\beta, p)$-semi-norms, and set $\mathbf{U}$ for the union of all $\mathbf{U}(\beta, p)$ 's.

Remarks. (a) Of course it suffices to take $n=2$ in the definition of $p$-superadditivity. We wrote the property in this form in order to make it symmetric with the $p$-subadditivity and the corresponding lower $(\beta, p)$-semi-norms, which will appear just below. 
(b) From the inequality $(a+b)^{q} \geqslant a^{q}+b^{q}$ for every $a, b \geqslant 0$ and $q \geqslant 1$, it follows that $\mathbf{U}(\beta, p) \subset \mathbf{U}\left(\beta, p^{\prime}\right)$ whenever $p^{\prime} \geqslant p$.

(c) In the definition of upper $(\beta, p)$-semi-norm, one can ask for the possible values of the parameters $\beta$ and $p$. By self-similarity and translation-invariance, it is easy to see that if $\mathbf{1} \in \mathcal{F}$ and $0<\|\mathbf{1}\|_{[0,1]}<\infty$ (where $\mathbf{1}$ stands for the unit function), then necessarily $\beta+1 / p \leqslant 0$. However, the examples (c)-(f) below show that this inequality is no longer true whenever $\|\mathbf{1}\|_{[0,1]}=0$.

We stress that most of the usual semi-norms belong to $\mathbf{U}$. In the following examples we always assume that $\mathcal{F}=\left\{f:\|f\|_{I}<\infty, I \in \mathcal{I}\right\}$.

Examples. (a) The supremum semi-norm, which is given by

$$
\|f\|_{I}=\sup _{t \in I}|f(t)|
$$

for every $I \in \mathcal{I}$, belongs to $\mathbf{U}(0,+\infty)$.

(b) The $L_{p}$-semi-norm, $p \geqslant 1$, which is given by

$$
\|f\|_{I}=\left(\int_{I}|f(s)|^{p} d s\right)^{1 / p}
$$

for every $I \in \mathcal{I}$, belongs to $\mathbf{U}(-1 / p, p)$.

(c) The $\eta$-Hölder semi-norm, $0 \leqslant \eta \leqslant 1$, which is given by

$$
\|f\|_{I}=\sup _{s<t \in I} \frac{|f(t)-f(s)|}{|t-s|^{\eta}}
$$

for every $I \in \mathcal{I}$, belongs to $\mathbf{U}(\eta,+\infty)$. In particular, the oscillation semi-norm $(\eta=0)$ belongs to $\mathbf{U}(0,+\infty)$. Similarly, the Calderón-Zygmund semi-norm

$$
\|f\|_{I}=\sup _{s<t \in I} \frac{|2 f((t+s) / 2)-f(s)-f(t)|}{(t-s)}
$$

belongs to $\mathbf{U}(1,+\infty)$, and the $\eta$-Lipschitz semi-norm, $\eta>1$, which is given by

$$
\|f\|_{I}=\sup _{s<t \in I} \frac{\left|f^{(n)}(t)-f^{(n)}(s)\right|}{|t-s|^{\eta-n}}
$$

where $n<\eta<n+1$ (and by its Calderón-Zygmund analogue for $\eta=n$ ), belongs to $\mathbf{U}(\eta,+\infty)$.

(d) The (strong) $p$-variation semi-norm, $p \geqslant 1$, which is given by

$$
\|f\|_{I}=\left(\sup _{t_{0}<\cdots<t_{n} \in I} \sum_{i=1}^{n}\left|f\left(t_{i}\right)-f\left(t_{i-1}\right)\right|^{p}\right)^{1 / p}
$$

for every $I \in \mathcal{I}$, belongs to $\mathbf{U}(0, p)$.

(e) The $(\eta, p)$-Sobolev semi-norm, $p \geqslant 1$ and $0 \leqslant \eta+1 / p<1$, which is given by

$$
\|f\|_{I}=\left(\iint_{I}\left(\frac{|f(t)-f(s)|}{|t-s|^{\eta+1 / p}}\right)^{p} d s d t\right)^{1 / p}
$$

for every $I \in \mathcal{I}$, belongs to $\mathbf{U}(\eta-1 / p, p)$.

(f) The $(\eta, p, g)$-Besov semi-norm, $\eta>0$ and $p, q \geqslant 1$, which is given by

$$
\|f\|_{I}=\left(\int_{0}^{|I|}\left(\frac{\omega_{p, I}(t, f)}{t^{\eta}}\right)^{q} \frac{d t}{t}\right)^{1 / q}
$$


for every $I \in \mathcal{I}$, where

$$
\omega_{p, I}(t, f)=\sup _{|h| \leqslant t}\left(\int_{I_{h}}|f(x-h)-f(x)|^{p} d x\right)^{1 / p},
$$

$I_{h}=\{x \in I \mid x-h \in I\}$ and $|I|$ stands for the Lebesgue measure of $I$, belongs to $\mathbf{U}(\eta-1 / p,+\infty)$. Notice that the usual $(\eta, p, q)$-Besov semi-norm, where the $L_{p}$-semi-norm is added in the definition (see e.g. [13]), does not belong to $\mathbf{U}$ since $L_{p}$-semi-norms have a different self-similarity index.

It follows from Remark (b) above that the concept of upper $(\beta, p)$-semi-norm is not sharp enough for our further purposes. Namely, the parameter $p$ which appears in our main Theorem 4 is not uniquely defined. Therefore, we need some kind of inverse property to (D), in order to work with a family of disjoint sets of semi-norms. Let $\mathcal{C}_{K}^{\ell}$ (resp. $\mathcal{C}_{K}^{0}$ ) denote the class of all $\ell$-times continuously differentiable (resp. continuous) functions with compact support. For technical reasons, we make the following assumptions on $\|\cdot\|$.

(E) $\mathcal{C}_{K}^{\ell} \subset \mathcal{F}$. ( $\ell$-smooth-finiteness)

(F) For every $I \in \mathcal{I}$ and $f, f_{n} \in \mathcal{F}_{I}$,

$$
f_{n} \rightarrow f \text { uniformly on } I \Rightarrow\|f\|_{I} \leqslant \lim \sup \left\|f_{n}\right\|_{I} \text {. (Lower semi-continuity) }
$$

Remark. Clearly, $\ell$-smooth-finiteness yields $\|f\|_{I}<+\infty$ for every $f$ of class $\mathcal{C}^{\ell}$ and every $I \in \mathcal{I}$.

Semi-continuity and $\ell$-smooth-finiteness (with appropriate $\ell$ depending on the semi-norm) are verified by all classical semi-norms.

Definition 2. Let $\beta \in \mathbb{R}, p \in(0,+\infty]$ and $\|\cdot\|$ be a contractive, translation-invariant, $\ell$-smooth-finite, lower semicontinuous and $\beta$-self-similar semi-norm on $\mathcal{F}$. We say that $\|\cdot\|$ is a lower $(\beta, p)$-semi-norm if it satisfies the following property:

(G) There exists $C_{p}>0$ such that for every $a_{0}<\cdots<a_{n} \in \mathbb{R}$ and $f \in C_{K}^{\ell}$ verifying $f\left(a_{0}\right)=\cdots=f\left(a_{n}\right)=0$,

$$
\begin{cases}\|f\|_{\left[a_{0}, a_{n}\right]} \leqslant C_{p}\left(\|f\|_{\left[a_{0}, a_{1}\right]}^{p}+\cdots+\|f\|_{\left[a_{n-1}, a_{n}\right]}^{p}\right)^{1 / p} & \text { if } p<+\infty, \quad \\ \|f\|_{\left[a_{0}, a_{n}\right]} \leqslant C_{\infty} \sup \left(\|f\|_{\left[a_{0}, a_{1}\right]}, \ldots,\|f\|_{\left[a_{n-1}, a_{n}\right]}\right) & \text { if } p=+\infty .\end{cases}
$$

In the following we will denote by $\mathbf{L}(\beta, p)$ the set of lower $(\beta, p)$-semi-norms, and set $\mathbf{L}$ for the union of the $\mathbf{L}(\beta, p)$ 's. We will say that $\|\cdot\|$ is a $(\beta, p)$-semi-norm if it belongs to $\mathbf{N}(\beta, p)=\mathbf{U}(\beta, p) \cap \mathbf{L}(\beta, p)$ and analogously we will set $\mathbf{N}$ for the union of $\mathbf{N}(\beta, p)$ 's.

Remarks. (a) Since the constant $C_{p}$ may be larger than 1, it is not enough to take $n=2$ in the definition of $p$-subadditivity, contrary to $p$-superadditivity.

(b) From the inequality $\left(a_{0}+\cdots+a_{n}\right)^{q} \leqslant a_{0}^{q}+\cdots+a_{n}^{q}$ for every $a_{0}, \ldots, a_{n} \geqslant 0$ and $0 \leqslant q \leqslant 1$, it follows that $\mathbf{L}(\beta, p) \subset \mathbf{L}\left(\beta, p^{\prime}\right)$ whenever $p^{\prime} \leqslant p$.

(c) Again, one can ask for the possible values of the parameters $\beta$ and $p$ in the definition of a lower $(p, \beta)$ semi-norm. By self-similarity and translation-invariance, it is easy to see that when the inequalities in (G) hold for the function $f=\mathbf{1}$ and for all partitions (assuming of course that $\mathbf{1} \in \mathcal{F}$ ) and if $0<\|\mathbf{1}\|_{[0,1]}<\infty$, then necessarily $\beta+1 / p \geqslant 0$. This inequality is actually true for all available examples, but we were unable to prove it in full generality.

(d) The supplementary assumption $f\left(a_{0}\right)=\cdots=f\left(a_{n}\right)=0$ makes sense, because in some cases $p$-subadditivity inequalities fail on more general functions (e.g. for $p$-variation norm and for Hölder norm). 
Notice that each of the above examples (a)-(d) belongs to $\mathbf{L}$, and hence to $\mathbf{N}$. Actually in each case we had chosen the smallest possible parameter $p$, i.e. we could have written $\|\cdot\| \in \mathbf{L}(\beta, p)$ as well as $\|\cdot\| \in \mathbf{U}(\beta, p)$. This fact is trivial for examples (a) and (b) where we can take $C_{p}=1$. In examples (c) and (d) we can take $C_{\infty}=2$ and $C_{p}=2^{1-1 / p}$ (as soon as $p \geqslant 1$ ), respectively. (Notice that in both cases the condition $f\left(a_{0}\right)=\cdots=f\left(a_{n}\right)=0$ is essential.)

However, $(\eta, p, q)$-Besov and $(\eta, p)$-Sobolev semi-norms do not belong to $\mathbf{L}$, because in these cases the constant $C_{p}$ may depend on the subdivision $a_{0}<\cdots<a_{n}$. These two examples are important in certain contexts, and this is why we would like to introduce a weaker definition, which will be given in terms of the evaluation of $\|\cdot\|$ along specific families of functions.

If $\ell \in \mathbb{N}$ and $\psi \in \mathcal{C}_{K}^{\ell}$, we introduce $\Psi=\left\{\psi_{j n}, n \in \mathbb{Z}, j \geqslant 0\right\}$, the two-parametric subset of $\mathcal{C}_{K}^{\ell}$ defined by

$$
\psi_{j n}(t)=\psi\left(2^{j}(t-n)\right)
$$

for every $n \in \mathbb{Z}, j \geqslant 0, t \in \mathbb{R}$.

Definition 3. Let $\beta \in \mathbb{R}, p \in(0,+\infty]$ and $\|\cdot\|$ be a lower semi-continuous, $\ell$-smooth-finite semi-norm on $\mathcal{F}$. We say that $\|\cdot\|$ is a lower $(\beta, p)$-semi-norm in the wide sense with respect to $\Psi$, if it satisfies the following property:

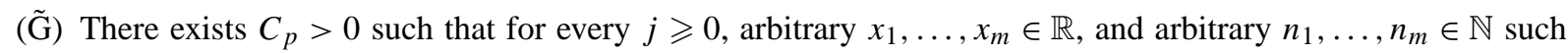
that the supports of the functions $\left\{\psi_{j, n_{i}}, 1 \leqslant i \leqslant m\right\}$ have disjoint interiors,

$$
\begin{cases}\left\|\sum_{i=1}^{m} x_{i} \psi_{j n_{i}}\right\|_{[0,1]} \leqslant C_{p} 2^{\beta j}\left(\sum_{i=1}^{m}\left|x_{i}\right|^{p}\right)^{1 / p} & \text { if } p<+\infty \\ \left\|\sum_{i=1}^{m} x_{i} \psi_{j n_{i}}\right\|_{[0,1]} \leqslant C_{\infty} 2^{\beta j} \sup _{1 \leqslant i \leqslant m}\left|x_{i}\right| & \text { if } p=+\infty\end{cases}
$$

Similarly we will denote by $\tilde{\mathbf{L}}(\beta, p)$ the set of lower $(\beta, p)$-semi-norms in the wide sense, and set $\tilde{\mathbf{L}}$ for the union of the $\tilde{\mathbf{L}}(\beta, p)$ 's. Analogously, we define $\tilde{\mathbf{N}}(\beta, p)=\mathbf{U}(\beta, p) \cap \tilde{\mathbf{L}}(\beta, p)$ and set $\tilde{\mathbf{N}}$ for the union of the $\tilde{\mathbf{N}}(\beta, p)$ 's.

Remarks. (a) In the sequel, $\Psi$ will be either a family of sufficiently smooth wavelet functions, or the Schauder system on $[0,1]$.

(b) Condition $(\tilde{G})$ means, in particular, that $(\mathrm{G})$ holds for linear combinations of specific functions with the same "frequency", and it is a well-known condition to obtain lower bounds for small deviation probabilities in a Gaussian framework [41,42]. Actually we will use it for the same purposes, but sometimes in a more general context, see Section 4.

(c) At first sight, neither translation-invariance nor self-similarity are required in the definition of $\tilde{\mathbf{L}}(\beta, p)$. Actually, working with a specific family of functions allows us to combine these necessary properties in the single inequality given by $(\tilde{\mathrm{G}})$.

(d) Again we can prove that $\mathbf{L}(\beta, p) \subset \mathbf{L}\left(\beta, p^{\prime}\right)$ whenever $p^{\prime} \leqslant p$.

(e) It is a bit tedious but not difficult to see that the $(\eta, p, q)$-Besov semi-norm belongs to $\tilde{\mathbf{N}}(\eta,+\infty)$ and that the $(\eta, p, q)$-Sobolev semi-norm belongs to $\tilde{\mathbf{N}}(\eta-1 / p, p)$, both with respect to the Schauder system.

Notation. In the following we will mainly consider functions restricted to $[0,1]$. By minor abuse of notations, and for the sake of brevity, we will set $\|f\|=\|f\|_{[0,1]}$ for every $f \in \mathcal{F}$, although such statements like " $\|\cdot\| \in \mathbf{U}(\beta, p)$ " or " $\|\cdot\| \in \mathbf{L}(\beta, p)$ " will always refer to the family $\|\cdot\|=\left\{\|\cdot\|_{I}, I \in \mathcal{I}\right\}$. By $C$ we will always mean a positive finite constant independent of the involved parameters, and whose value may change from line to line. 


\subsection{Riemann-Liouville processes and their associated linear fractional stable motions}

Let $\left\{Z_{t}, t \geqslant 0\right\}$ be a symmetric $\alpha$-stable process with index $\alpha \in(0,2]$, i.e. $Z$ is a Lévy process whose characteristic function is given by

$$
\mathbb{E}\left[e^{i \lambda Z_{t}}\right]=e^{-t|\lambda|^{\alpha}}
$$

for every $t \geqslant 0$ and $\lambda \in \mathbb{R}$. It is well known and easy to see that for every $H>0$ the following stochastic integral:

$$
R_{t}^{H}=\int_{0}^{t}(t-s)^{H-1 / \alpha} d Z_{s},
$$

is well-defined for every $t>0$. We set $R_{0}^{H}=0$ and call $\left\{R_{t}^{H}, t \geqslant 0\right\}$ the Riemann-Liouville process (in abridged form: RLP) with Hurst parameter $H$. This latter terminology is motivated by the following $H$-self-similarity property of $R^{H}$ : for every $c>0$

$$
\left\{R_{c t}^{H}, t \geqslant 0\right\} \stackrel{d}{=}\left\{c^{H} R_{t}^{H}, t \geqslant 0\right\} .
$$

When no confusion is possible, we will drop the subscript $H$ and write $R=R^{H}$ for the sake of brevity. Notice that $R$ has no stationary increments, unless $H=1 / \alpha$. Instead of this, $R$ has some kind of extrapolation-homogeneity, which will be important in the sequel: namely, if we set

$$
R_{a, t}=\int_{a}^{t}(t-s)^{H-1 / \alpha} d Z_{s}
$$

for every $t \geqslant a \geqslant 0$, then the following equality in law holds:

$$
\left\{R_{a, a+t}, t \geqslant 0\right\} \stackrel{d}{=}\left\{R_{t}, t \geqslant 0\right\} .
$$

Moreover, $R$ has an equally important independence property: if $\tilde{R}_{a, t}=R_{t}-R_{a, t}$, then for every $a \geqslant 0$ the processes $\left\{R_{a, a+t}, t \geqslant 0\right\}$ and $\left\{\tilde{R}_{a, a+t}, t \geqslant 0\right\}$ are independent. These three properties follow easily from selfsimilarity, stationarity and independence of the increments of $Z$.

Of course, $Z$ itself is an RLP with Hurst parameter $H=1 / \alpha$. Notice also that up to normalization constants, the family of Riemann-Liouville processes

$$
\left\{R^{H}, H \geqslant \max \{0,1 / \alpha-1\}\right\}
$$

is closed with respect to time-integration. In particular, the $m$-times integrated Brownian motion is an RLP with parameters $\alpha=2$ and $H=m+1 / 2$.

The Riemann-Liouville process is closely related to $\left\{X_{t}^{H}, t \geqslant 0\right\}$, the so-called linear stable fractional motion (in abridged form: LFSM) with Hurst parameter $H . X^{H}$ can be defined through the following (independent) decomposition: $X^{H}=R^{H}+M^{H}$ where $M_{0}^{H}=0$ and

$$
M_{t}^{H}=\int_{0}^{+\infty}\left((t+s)^{H-1 / \alpha}-s^{H-1 / \alpha}\right) d \tilde{Z}_{s}
$$

for every $t>0, \tilde{Z}$ being an independent copy of $Z$. We call $M^{H}$ the long memory process (in abridged form: LMP) associated to $X^{H}$. We use the notion of "long memory" in a non-standard sense here (long memory is nowadays commonly related to a certain range of $H$ ), since it perfectly reflects the unboundedness of the support of the corresponding kernel, contrary to that of RLP. 
Notice that the stochastic integral defining $M^{H}$ diverges a.s. at $+\infty$ as soon as $H \geqslant 1$. Hence, $X^{H}$ can be defined only for $H \in(0,1)$. Again, when no confusion is possible, we will drop the subscript $H$ and write $X=X^{H}$ (resp. $M=M^{H}$ ) for the sake of brevity.

$X$ is also $H$-self-similar and its increments are stationary:

$$
\left\{X_{s+t}-X_{s}, t \geqslant 0\right\} \stackrel{d}{=}\left\{X_{t}, t \geqslant 0\right\}
$$

for every $s \geqslant 0$, and $X$ is a so-called $H$-sssi process. We refer to the monographs [18,36] for an extensive account on these latter processes. Notice that in the Gaussian case $\alpha=2, X$ coincides with the canonical fractional Brownian motion, up to some numerical factor (see Proposition 7.2.6 in [36]). Moreover, an alternative definition of $X$ can then be given through the following ("well-balanced") decomposition:

$$
X_{t}=\int_{-\infty}^{+\infty}\left(|t+s|^{H-1 / 2}-|s|^{H-1 / 2}\right) \dot{B}(d s),
$$

where $\dot{B}$ is the usual white noise (see Exercise 7.2 in [36]). When $\alpha<2$, the corresponding integral

$$
\tilde{X}_{t}=\int_{-\infty}^{+\infty}\left(|t+s|^{H-1 / \alpha}-|s|^{H-1 / \alpha}\right) \dot{Z}(d s)
$$

also makes sense, where $\dot{Z}$ is the so-called symmetric $\alpha$-stable noise. However, $\tilde{X}$ is no more equivalent to $X$ (see Theorem 7.4.5 in [36]). Actually, many other "bilateral" $H$-sssi $\alpha$-stable processes can be constructed, which are all non-equivalent except in the Gaussian case (see Definition 7.4.1 and Theorem 7.4.5 in [36]). Hence, our unilateral LFSM process is just one possible stable generalization of fractional Brownian motion. However, we will restrict our study to this unilateral process, even though our results can probably be adapted to some other situations.

Notice that the process $M$ is smooth on $(0,+\infty)$, so that $X$ and $R$ exhibit similar local properties. In particular it is well known (see Chapters 9-12 in [36]) that $R$ (resp. $X$ ) admits a continuous version if and only if

$$
\alpha=2 \text { or } H>1 / \alpha .
$$

In Section 6 we will give some other local properties of $R$ and $X$, related to the examples of semi-norms (a)(f) listed above. We finally refer to [30] for a more thorough comparison between $R$ and $X$, in the context of econometric applications.

\section{Existence of the small deviation constant for RLP}

We can now state the main result of this paper.

Theorem 4. Let $\|\cdot\| \in \mathbf{U}(\beta, p)$ and $R$ be an RLP with Hurst parameter $H$. Assume that $H>\beta+1 / p$ and set $\gamma=(H-\beta-1 / p)^{-1}$. Then there exists $\mathcal{K} \in(0,+\infty]$ such that

$$
\lim _{\varepsilon \downarrow 0} \varepsilon^{\gamma} \log \mathbb{P}[\|R\| \leqslant \varepsilon]=-\mathcal{K} .
$$

Remarks. (a) It is interesting to note that the stability index $\alpha$ does not directly show up in the expression of the small deviation rate.

(b) This result says nothing about the finiteness of the constant $\mathcal{K}$, which is of course a very important feature. To this aim, one needs to show a lower bound for small deviation probabilities with appropriate order - see Section 4 . 
While a priori nothing indicates that the order $\gamma$ of small deviation probabilities we propose in Theorem 4 is the right one, it is indeed correct for all available examples, as soon as $p$ is chosen as small as possible - see Section 6 .

The next two paragraphs are devoted to the proof of Theorem 4 and we fix once and for all an upper $(\beta, p)$ semi-norm $\|\cdot\|$, and an RLP $R$ with Hurst parameter $H>\beta+1 / p$. We begin with proving a kind of stochastic superadditivity property, which will be our crucial argument.

\subsection{Stochastic superadditivity}

Let $X$ and $Y$ be two real-valued random variables. We say that $X$ is stochastically larger than $Y$ if and only if

$$
\mathbb{P}\{X \leqslant r\} \leqslant \mathbb{P}\{Y \leqslant r\}
$$

for every $r \in \mathbb{R}$, and we write in this case $X \succeq Y$. If $X$ and $Y$ are positive, then clearly $X \succeq Y$ entails that

$$
\mathbb{E}[\exp -\lambda X] \leqslant \mathbb{E}[\exp -\lambda Y]
$$

for every $\lambda \geqslant 0$.

Proposition 5. Suppose that $p<\infty$. Let $R_{1}, R_{2}$ be two independent copies of $R$ and set $q=p(H-\beta)>0$. Then for every $a, b \geqslant 0$

$$
(a+b)^{q}\|R\|^{p} \succeq a^{q}\left\|R_{1}\right\|^{p}+b^{q}\left\|R_{2}\right\|^{p} .
$$

Proof. Fix $a, b>0$ and set $c=a+b$. We first use the $p$-superadditivity of $\|\cdot\|$ and get

$$
\|R\|_{[0, c]}^{p} \geqslant\|R\|_{[0, a]}^{p}+\|R\|_{[a, c]}^{p} \text {. }
$$

By self-similarity for both $\|\cdot\|$ and $R$, we also have

$$
\|R\|_{[0, c]}^{p} \stackrel{d}{=}\left\|c^{H} R\left(c^{-1} \cdot\right)\right\|_{[0, c]}^{p}=c^{p(H-\beta)}\|R\|_{[0,1]}^{p}=c^{q}\|R\|^{p}
$$

and, similarly,

$$
\|R\|_{[0, a]}^{p} \stackrel{d}{=} a^{q}\left\|R_{1}\right\|^{p} \quad \text { and } \quad\|R\|_{[0, b]}^{p} \stackrel{d}{=} b^{q}\left\|R_{2}\right\|^{p} .
$$

Putting everything together, we now see that it is enough to show

$$
\|R\|_{[0, a]}^{p}+\|R\|_{[a, c]}^{p} \succeq\|R\|_{[0, a]}^{p}+\left\|R_{a}\right\|_{[a, c]}^{p},
$$

because of the translation-invariance of $\|\cdot\|$ and the extrapolation-homogeneity of $R$. Here and throughout this section, $R_{a}$ denotes the process $\left\{R_{a, t}, t \geqslant a\right\}$. For every $a \geqslant 0$, set $\mathcal{G}_{a}$ for the $\sigma$-field generated by $\left\{Z_{s}, 0 \leqslant s \leqslant a\right\}$ and $\mathbb{P}_{a}$ for the conditional probability with respect to $\mathcal{G}_{a}$. We clearly have

$$
\begin{aligned}
\mathbb{P}\left[\|R\|_{[0, a]}^{p}+\|R\|_{[a, c]}^{p} \leqslant r\right] & =\mathbb{E}\left[\mathbb{P}_{a}\left[\|R\|_{[a, c]}^{p} \leqslant r-\|R\|_{[0, a]}^{p}\right]\right] \\
& =\mathbb{E}\left[\mathbb{P}_{a}\left[\left\|\tilde{R}_{a}+R_{a}\right\|_{[a, c]}^{p} \leqslant r-\|R\|_{[0, a]}^{p}\right]\right]
\end{aligned}
$$

for every $r \in \mathbb{R}$. Since $R_{a}$ is a conditionally Gaussian process under $\mathbb{P}_{a}$ (see [36, pp. 153-154]), we can apply Anderson's inequality under $\mathbb{P}_{a}$ and get (recall that $\tilde{R}_{a}$ is $\mathcal{G}_{a}$-measurable)

$$
\mathbb{P}_{a}\left[\left\|\tilde{R}_{a}+R_{a}\right\|_{[a, c]}^{p} \leqslant r-\|R\|_{[0, a]}^{p}\right] \leqslant \mathbb{P}_{a}\left[\left\|R_{a}\right\|_{[a, c]}^{p} \leqslant r-\|R\|_{[0, a]}^{p}\right] .
$$

Now, since $R_{a}$ is independent of $\mathcal{G}_{a}$, we can average backwards and obtain

$$
\mathbb{P}\left[\|R\|_{[0, a]}^{p}+\|R\|_{[a, c]}^{p} \leqslant r\right] \leqslant \mathbb{P}\left[\|R\|_{[0, a]}^{p}+\left\|R_{a}\right\|_{[a, c]}^{p} \leqslant r\right]
$$

for every $r \in \mathbb{R}$, as desired for (5). 
The next proposition covers the simpler situation when $p=\infty$, and follows mainly the outline of Theorem 2.1 in [23].

Proposition 6. Suppose that $p=+\infty$. Let $R_{1}, R_{2}$ be two independent copies of $R$ and set $q=H-\beta>0$. Then for every $a, b \geqslant 0$

$$
(a+b)^{q}\|R\| \succeq \max \left\{a^{q}\left\|R_{1}\right\| ; b^{q}\left\|R_{2}\right\|\right\} .
$$

Proof. We need to show that for all $r \in \mathbb{R}$

$$
\mathbb{P}\left[(a+b)^{q}\|R\| \leqslant r\right] \leqslant \mathbb{P}\left[a^{q}\|R\| \leqslant r\right] \mathbb{P}\left[b^{q}\|R\| \leqslant r\right] .
$$

Set $c=a+b$. Again we use the $\infty$-superadditivity of $\|\cdot\|$ and get

$$
\|R\|_{[0, c]} \geqslant \sup \left\{\|R\|_{[0, a]},\|R\|_{[a, c]}\right\} .
$$

Therefore,

$$
\mathbb{P}\left[\|R\|_{[0, c]} \leqslant r\right] \leqslant \mathbb{P}\left[\|R\|_{[0, a]} \leqslant r,\|R\|_{[a, c]} \leqslant r\right]=\mathbb{E}\left[\mathbf{1}_{\left\{\|R\|_{[0, a]} \leqslant r\right\}} \mathbb{P}_{a}\left[\|R\|_{[a, c]} \leqslant r\right]\right]
$$

for every $r \in \mathbb{R}$, where $\mathbb{P}_{a}$ is defined as above. By Anderson's inequality, independence of $R_{a}$ of $\mathcal{G}_{a}$, extrapolationhomogeneity of $R$ and translation-invariance of $\|\cdot\|$, we obtain

$$
\mathbb{P}_{a}\left[\|R\|_{[a, c]} \leqslant r\right]=\mathbb{P}_{a}\left[\left\|\tilde{R}_{a}+R_{a}\right\|_{[a, c]} \leqslant r\right] \leqslant \mathbb{P}_{a}\left[\left\|R_{a}\right\|_{[a, c]} \leqslant r\right]=\mathbb{P}\left[\|R\|_{[0, b]} \leqslant r\right] .
$$

Averaging backwards, we get

$$
\mathbb{P}\left[\|R\|_{[0, c]} \leqslant r\right] \leqslant \mathbb{P}\left[\|R\|_{[0, a]} \leqslant r\right] \mathbb{P}\left[\|R\|_{[0, b]} \leqslant r\right]
$$

for every $r \in \mathbb{R}$. Now this yields the desired inequality

$$
\mathbb{P}\left[c^{q}\|R\| \leqslant r\right] \leqslant \mathbb{P}\left[a^{q}\|R\| \leqslant r\right] \mathbb{P}\left[b^{q}\|R\| \leqslant r\right],
$$

since by self-similarity of $R$ and $\|\cdot\|$

$$
\mathbb{P}\left[\|R\|_{[0, t]} \leqslant r\right]=\mathbb{P}\left[t^{q}\|R\| \leqslant r\right]
$$

for every $t \geqslant 0$ and $r \in \mathbb{R}$.

We are now ready to proceed to the proof of Theorem 4.

\subsection{Proof of Theorem 4}

Again we first consider the case $p<\infty$. Proposition 5 yields the following decisive inequality for Laplace transforms:

$$
\mathbb{E}\left[\exp -(a+b)^{q}\|R\|^{p}\right] \leqslant \mathbb{E}\left[\exp -a^{q}\|R\|^{p}\right] \mathbb{E}\left[\exp -b^{q}\|R\|^{p}\right]
$$

for every $a, b \geqslant 0$ and $q=p(H-\beta)$ as above. This entails that the function $\Phi$ defined by

$$
\Phi(h)=\log \mathbb{E}\left[\exp -h^{q}\|R\|^{p}\right]
$$

for every $h \geqslant 0$, is a continuous negative function which satisfies $\Phi(a+b) \leqslant \Phi(a)+\Phi(b)$ for every $a, b \geqslant 0$. By the standard subadditivity argument, we obtain

$$
\lim _{h \rightarrow \infty} \frac{\Phi(h)}{h}=\inf _{h \geqslant 0} \frac{\Phi(h)}{h}=-C \in[-\infty, 0)
$$


and, returning to the Laplace transform,

$$
\lim _{\lambda \rightarrow+\infty} \lambda^{1 / q} \log \mathbb{E}\left[\exp -\lambda\|R\|^{p}\right]=-C .
$$

Notice that $q>1$ by assumption. Hence we can apply de Bruijn's exponential Tauberian theorem (see Theorem 4.12.9 in [6], or Theorem 3.5 in [26] for a more comfortable formulation), which yields

$$
\lim _{\varepsilon \rightarrow 0} \varepsilon^{1 /(q-1)} \log \mathbb{P}\left[\|R\|^{p} \leqslant \varepsilon\right]=-\mathcal{K}=-(q-1)(C / q)^{q /(q-1)},
$$

and finally

$$
\lim _{\varepsilon \rightarrow 0} \varepsilon^{\gamma} \log \mathbb{P}[\|R\| \leqslant \varepsilon]=-\mathcal{K} \in[-\infty, 0)
$$

with $\gamma=(H-\beta-1 / p)^{-1}$. This completes the proof of Theorem 4 when $p<+\infty$.

In the case $p=+\infty$ we do not even need Laplace transform, since it follows directly from Proposition 6 that the function

$$
\Phi(h)=\log \mathbb{P}\left[\|R\| \leqslant h^{\beta-H}\right]
$$

is subadditive. Again this entails

$$
\lim _{h \rightarrow \infty} \frac{\Phi(h)}{h}=\inf _{h \geqslant 0} \frac{\Phi(h)}{h}=-\mathcal{K} \in[-\infty, 0),
$$

and we obtain

$$
\lim _{\varepsilon \rightarrow 0} \varepsilon^{\gamma} \log \mathbb{P}[\|R\| \leqslant \varepsilon]=-\mathcal{K} \in[-\infty, 0)
$$

with $\gamma=(H-\beta)^{-1}$, as desired when $p=+\infty$.

\section{Lower bounds: finiteness of the constant for continuous RLP}

In this section we obtain a suitable lower bound for small deviation probabilities which will allow us to prove, under certain conditions, that the constant $\mathcal{K}$ from Theorem 4 is actually finite whenever $\|\cdot\| \in \mathbf{L}(\beta, p)$ as well. Unfortunately, our method is only efficient in the continuous case, i.e. when

$$
\alpha=2 \text { or } H>1 / \alpha .
$$

An explanation for this understandable, but important restriction on $R$ will be given later. Our result reads as follows:

Theorem 7. Let $\|\cdot\| \in \mathbf{L}(\beta, p)$ and $R$ be a continuous $\alpha$-stable RLP with Hurst parameter $H$. Suppose that $H>\beta+1 / p$ if $\alpha=2$ and $H>\beta+1 / p+1 / \alpha$ if $\alpha<2$. Then

$$
\liminf _{\varepsilon \downarrow 0} \varepsilon^{\gamma} \log \mathbb{P}[\|R\| \leqslant \varepsilon]>-\infty,
$$

with $\gamma=(H-\beta-1 / p)^{-1}$.

Combining Theorem 4 and Theorem 7 yields readily the following fairly general small deviation theorem for continuous Riemann-Liouville processes: 
Corollary 8. Let $\|\cdot\| \in \mathbf{N}(\beta, p)$ and $R$ be a continuous $\alpha$-stable RLP with Hurst parameter H. Suppose that $H>\beta+1 / p$ if $\alpha=2$ and $H>\beta+1 / p+1 / \alpha$ if $\alpha<2$. Then there exists $\mathcal{K} \in(0, \infty)$ such that

$$
\lim _{\varepsilon \downarrow 0} \varepsilon^{\gamma} \log \mathbb{P}[\|R\| \leqslant \varepsilon]=-\mathcal{K},
$$

with $\gamma=(H-\beta-1 / p)^{-1}$.

We stress that if the parameter $H$ is not too large, more precisely if $H<2$, then it is possible to obtain the lower bound for small deviation probabilities under a weaker assumption than $\|\cdot\| \in \mathbf{L}(\beta, p)$. Here we just need that $\|\cdot\| \in \tilde{\mathbf{L}}(\beta, p)$ with respect to

$$
\Psi=\left\{\psi_{j n}=\psi\left(2^{j} t-n+1\right), 1 \leqslant n \leqslant 2^{j}, j \geqslant 0\right\},
$$

the Schauder system generated by the triangular function $\psi(t)=\mathbf{1}_{[0,1]}(t)(1-|2 t-1|)$.

Notice that on each level $j$ the supports of the functions $\psi_{j n}$ have disjoint interiors, so that here $\|\cdot\| \in \tilde{\mathbf{L}}(\beta, p)$ w.r.t. $\Psi$ simply means that

$$
\left\|\sum_{n=1}^{2^{j}} x_{n} \psi_{j n}\right\| \leqslant C_{p} 2^{\beta j}\left(\sum_{n=1}^{2^{j}}\left|x_{n}\right|^{p}\right)^{1 / p}
$$

for $p<+\infty$, with an obvious modification for $p=\infty$. We have an analogous result to Theorem 7 :

Theorem 9. Let $\|\cdot\| \in \tilde{\mathbf{L}}(\beta, p)$ w.r.t. Schauder system and $R$ be a continuous $\alpha$-stable RLP with Hurst parameter $H<2$. Suppose that $H>\beta+1 / p$ if $\alpha=2$ and $H>\beta+1 / p+1 / \alpha$ if $\alpha<2$. Then

$$
\liminf _{\varepsilon \downarrow 0} \varepsilon^{\gamma} \log \mathbb{P}[\|R\| \leqslant \varepsilon]>-\infty,
$$

with $\gamma=(H-\beta-1 / p)^{-1}$.

And, of course, we get the corresponding corollary:

Corollary 10. Let $\|\cdot\| \in \tilde{\mathbf{N}}(\beta, p)$ w.r.t. Schauder system and $R$ be a continuous $\alpha$-stable RLP with Hurst parameter $H<2$. Suppose that $H>\beta+1 / p$ if $\alpha=2$ and $H>\beta+1 / p+1 / \alpha$ if $\alpha<2$. Then there exists $\mathcal{K} \in(0, \infty)$ such that

$$
\lim _{\varepsilon \downarrow 0} \varepsilon^{\gamma} \log \mathbb{P}[\|R\| \leqslant \varepsilon]=-\mathcal{K},
$$

with $\gamma=(H-\beta-1 / p)^{-1}$.

Remark. The assumption $H<2$ is rather restrictive and this is why the Schauder basis is not always a sufficient tool. For example, if $\alpha<1 / 2$, then $H<2$ is incompatible with the continuity assumption $H>1 / \alpha$, thus for small $\alpha$ we get nothing from Theorem 9; recall however that Theorem 7 still works.

\subsection{Decorrelating stable arrays}

In this paragraph we prove a crucial lower bound, which is a generalization of Lemma 2.1 in [42] to arrays of symmetric $\alpha$-stable random variables, and which will be useful for both Theorems 7 and 9 . It relies on a version of Šidák's inequality for stable variables recently obtained in this framework by G. Samorodnitsky [35]. 
Lemma 11. Let $M, h>0$ and $\left\{y_{j n}, 1 \leqslant n \leqslant M 2^{h j}, j \geqslant 0\right\}$ be an array of identically distributed symmetric jointly $\alpha$-stable random variables. Let $z, \delta>0$ be such that $\delta<z$ if $\alpha=2$ and $\delta<z-h / \alpha$ if $\alpha<2$. Let $m>0$ be an integer and set

$$
d_{j}=d_{j}(m)=2^{z(j-m)-\delta|j-m|} .
$$

Then there exists a constant $C$ depending only on $M, h, z, \delta$ such that

$$
\mathbb{P}\left[\left|y_{j n}\right| \leqslant d_{j}, 1 \leqslant n \leqslant M 2^{h j}, j \geqslant 0\right] \geqslant \exp -C 2^{h m} .
$$

Proof. Up to normalization, the case $\alpha=2$ is just the statement of Lemma 2.1 in [42]. Hence we can concentrate on the case $\alpha<2$, and first notice that Lemma 2.1 in [35] entails the following decorrelation inequality:

$$
\mathbb{P}\left[\left|y_{j n}\right| \leqslant d_{j}, 1 \leqslant n \leqslant M 2^{h j}, j \geqslant 0\right] \geqslant \prod_{j \geqslant 0} \mathbb{P}\left[|y| \leqslant d_{j}\right]^{M 2^{h j}},
$$

where $y$ is some symmetric $\alpha$-stable random variable. We can decompose the right-hand side into

$$
\prod_{j \geqslant 0} \mathbb{P}\left[|y| \leqslant d_{j}\right]^{M 2^{h j}}=\left(\prod_{j \geqslant m} \mathbb{P}\left[|y| \leqslant d_{j}\right]^{M 2^{h j}}\right)\left(\prod_{j=0}^{m-1} \mathbb{P}\left[|y| \leqslant d_{j}\right]^{M 2^{h j}}\right) .
$$

To estimate the infinite product, we use the following well-known tail behavior of $y$ (see e.g. Property 1.2.15 in [36]):

$$
\lim _{r \uparrow+\infty} r^{\alpha} \mathbb{P}[|y|>r]=K_{1} \in(0,+\infty) .
$$

This yields

$$
\begin{aligned}
\log \prod_{j \geqslant m} \mathbb{P}\left[|y| \leqslant d_{j}\right]^{M 2^{h j}} & =M \sum_{j \geqslant m} 2^{h j} \log \left(1-\mathbb{P}\left[|y|>d_{j}\right]\right) \\
& \geqslant-C \sum_{j \geqslant m} 2^{h j} \mathbb{P}\left[|y|>2^{(z-\delta)(j-m)}\right] \\
& \geqslant-C \sum_{j \geqslant m} 2^{h j} 2^{-\alpha(z-\delta)(j-m)} \\
& \geqslant-C 2^{h m},
\end{aligned}
$$

where in the last inequality we used $h-\alpha(z-\delta)<0$. The estimate of the finite product is even simpler. Since $y$ has a positive density in the neighbourhood of the origin, we have

$$
\lim _{\varepsilon \downarrow 0} \varepsilon^{-1} \mathbb{P}[|y| \leqslant \varepsilon]=K_{2} \in(0,+\infty) .
$$

This entails

$$
\begin{aligned}
\log \prod_{j=0}^{m-1} \mathbb{P}\left[|y| \leqslant d_{j}\right]^{M 2^{h j}} & =M \sum_{j=0}^{m-1} 2^{h j} \log \mathbb{P}\left[|y| \leqslant d_{j}\right] \\
& \geqslant-C \sum_{j=0}^{m-1} 2^{h j}\left(1+\log \left(2^{(z+\delta)(m-j)}\right)\right) \\
& \geqslant-C 2^{h m} \sum_{j=0}^{m-1}(m-j) 2^{h(j-m)} \\
& \geqslant-C 2^{h m},
\end{aligned}
$$


where in the last inequality we used $h>0$. Putting everything together now yields

$$
\mathbb{P}\left[\left|y_{j n}\right| \leqslant d_{j}, 1 \leqslant n \leqslant M 2^{h j}, j \geqslant 0\right] \geqslant \exp -C 2^{h m}
$$

for a constant $C$ not depending on $m$.

Remark. In the non-Gaussian case, it is easy to see that the condition $\delta<z-h / \alpha$ is also necessary, because of the heavy tails of $\alpha$-stable random variables: indeed, if the $y_{j n}$ 's are mutually independent and if $z=\delta+h / \alpha$, then it follows from (7) that

$$
\mathbb{P}\left[\left|y_{j n}\right| \leqslant d_{j}, 1 \leqslant n \leqslant M 2^{h j}, j \geqslant 0\right]=0 .
$$

\subsection{Some elements of wavelet theory}

The proof of Theorem 7 relies mainly on a suitable wavelet decomposition of $R$, which we recall here for the sake of completeness. In this paragraph we fix once and for all a semi-norm $\|\cdot\| \in \mathbf{L}(\beta, p)$ which is $\ell$-smooth-finite, and $R$ a continuous RLP satisfying the assumptions of Theorem 7.

There exist two functions $\varphi, \psi \in \mathcal{C}_{K}^{\ell}$ ) (“wavelet parents") such that $\psi$ has vanishing moments up to order $\ell$ :

$$
\int_{-\infty}^{\infty} t^{k} \psi(t) d t=0, \quad 0 \leqslant k \leqslant \ell,
$$

and such that the wavelet functions $\left\{\psi_{j n}, n \in \mathbb{Z}, j \geqslant 1\right\}$ and $\left\{\varphi_{n}, n \in \mathbb{Z}\right\}$, respectively defined by

$$
\psi_{j n}(t)=2^{j / 2} \psi\left(2^{j} t-n\right) \text { and } \varphi_{n}(t)=\varphi(t-n),
$$

form an orthogonal base of $L_{2}(\mathbb{R})$. We refer to Daubechies' construction [15], Section 6.4 for the definition and Section 7.1 for smoothness properties of these compactly supported wavelets. A useful book is also [31].

Fix $[-D, D]$ an interval containing the supports of $\psi$ and $\varphi$. Consider $I$, the integration operator on compactly supported functions:

$$
I f(t)=\int_{-\infty}^{t} f(s) d s
$$

for every such $f$ and $t \in \mathbb{R}$. We set $I^{0}$ for the identity operator and $I^{k}$ for the $k$ th iteration of $I, k \geqslant 1$. Since for every $k \geqslant 0$

$$
I^{k+1} f(t)=\frac{1}{k !} \int_{-\infty}^{t} f(s)(t-s)^{k} d s,
$$

the moment condition (8) on $\psi$ entails that the functions $I^{k} \psi$ are also supported by the interval $[-D, D]$ for $0 \leqslant k \leqslant \ell$. In particular,

$$
I^{k} \psi( \pm D)=0, \quad 0 \leqslant k \leqslant \ell .
$$

Since under our assumptions $R$ is continuous, the process $\mathbf{1}_{[0,2 D+1]} R$ belongs a.s. to $L_{2}(\mathbb{R})$ (actually a weaker assumption $H>1 / \alpha-1 / 2$ would suffice here, see [36, Chapter 11]). Hence, we can write its wavelet decomposition:

$$
\mathbf{1}_{[0,2 D+1]} R=\sum_{j \geqslant 1} \sum_{n} r_{j n} \psi_{j n}+\sum_{n} r_{n} \varphi_{n},
$$


where

$$
r_{j n}=\int_{0}^{2 D+1} R(s) \psi_{j n}(s) d s \text { and } r_{n}=\int_{0}^{2 D+1} R(s) \varphi_{n}(s) d s .
$$

Actually more can be said about the convergence of the series on the right-hand side in (10). Namely, again from our assumption on $H$, it is well-known that $R$ is locally $\eta$-Hölder for some $\eta>0[20,43]$, and in particular the function $R$ coincides on $[-D, 3 D+1]$ with an $L_{2}$-function which is globaly $\eta$-Hölder. We know then (see e.g. Theorem 7 in [31, Chapter 6]) that the series (10) converges to $R$ uniformly on $[0,1]$.

Besides, in (10) we can delete each $\psi_{j n}$ and $\varphi_{n}$ whose support does not overlap with [0,1], and the remaining series still converges to $R$ uniformly on $[0,1]$. More precisely, for $j \geqslant 1$ let $N_{j}$ be the set of all positive integers $n$ such that the support of $\psi_{j n}$ overlaps with [0,1]. Similarly, let $N_{0}$ denote the set of all positive integers $n$ such that the support of $\varphi_{n}$ overlaps with $[0,1]$. We have, uniformly on $[0,1]$,

$$
R=\sum_{j \geqslant 1}\left(\sum_{n \in N_{j}} r_{j n} \psi_{j n}\right)+\sum_{n \in N_{0}} r_{n} \varphi_{n}
$$

We will need two elementary properties of the family $\left\{N_{j}\right\}$. First, since $\varphi$ and $\psi$ have compact support, we have an exponential bound of the array's size: there exists a constant $M$ depending only on $D$ such that for all $j$

$$
\sup _{n \in N_{j}}|n| \leqslant M 2^{j} .
$$

Second, for all $j \geqslant 0$ and all $n \in N_{j}$ the support of $\psi_{j n}$ overlaps with $[0,1]$ and its length is bounded by $2 D$. Hence, $\psi_{j n}$ vanishes on $[2 D+1, \infty)$ and we have

$$
\mathbf{1}_{[0, \infty)} \psi_{j n}=\mathbf{1}_{[0,2 D+1]} \psi_{j n}, \quad j \geqslant 0, n \in N_{j} .
$$

\subsection{Proof of Theorem 7}

We may (and will) assume that $\ell>H$. For every $n \in \mathbb{Z}$ we will set $\psi_{0 n}=\varphi_{n}$ and $r_{0 n}=r_{n}$ for the sake of concision. By lower semi-continuity and triangle's inequality, we clearly have

$$
\|R\|=\sum_{j \geqslant 0}\left\|\sum_{n \in N_{j}} r_{j n} \psi_{j n}\right\| .
$$

Moreover, since the parent functions have support in $[-D, D]$, for each $j \geqslant 0$ we can split the family $\left\{\psi_{j n}, n \in N_{j}\right\}$ into $[2 D]+1$ subfamilies such that in each subfamily the functions have supports with disjoint interiors. Suppose first that $p<+\infty$. The triangle inequality, condition $(\mathrm{G})$ and (11) yield

$$
\|R\| \leqslant C \sum_{j \geqslant 0}\left(\sum_{n \in N_{j}}\left|r_{j n}\right|^{p}\left\|\psi_{j n}\right\|^{p}\right)^{1 / p} .
$$

On the one hand, by translation-invariance, $\beta$-self-similarity, contractivity and $\ell$-smooth-finiteness of $\|\cdot\|$, it is easy to see that for every $j, n$,

$$
\left\|\psi_{j n}\right\| \leqslant C 2^{(1 / 2+\beta) j}
$$

for some constant $C$ independent of $j, n$. On the other hand, we can evaluate the coefficients as follows. Starting with the definition of $r_{j n}$ and making use of (12) we have for all $j \geqslant 1, n \in N_{j}$ 


$$
\begin{aligned}
r_{j n} & =\int_{0}^{2 D+1} R(s) \psi_{j n}(s) d s=\int_{0}^{+\infty} R(s) \psi_{j n}(s) d s \\
& =2^{j / 2} \int_{0}^{+\infty} R(s) \psi\left(2^{j} s-n\right) d s=2^{-j / 2} \int_{0}^{+\infty} R\left(2^{-j} s\right) \psi(s-n) d s \\
& \stackrel{d}{=} 2^{-(H+1 / 2) j} \int_{0}^{+\infty} R(s) \psi(s-n) d s=2^{-(H+1 / 2) j} \int_{\mathbb{R}} R(s+n) \psi(s) d s,
\end{aligned}
$$

where in the penultimate inequality we used the $H$-self-similarity of $R$. Plugging (14) and (15) into (13) and using (11) yields

$$
\|R\| \leqslant C \sum_{j \geqslant 0} 2^{-(H-\beta-1 / p) j}\left(\sup _{|n| \leqslant M 2^{j}}\left|r_{j n}^{\prime}\right|\right)
$$

where $r_{j n}^{\prime}$ stand for the renormalized wavelet coefficients of $R$, viz.

$$
r_{0 n}^{\prime}=r_{0 n} \quad \text { and } \quad r_{j n}^{\prime} \stackrel{d}{=} \int_{\mathbb{R}} R(s+n) \psi(s) d s \quad \text { if } j \geqslant 1 .
$$

Notice that one can write the wavelet coefficients from (17) in the following integral form: if $j \geqslant 1$, then $r_{j n}^{\prime}=$ $\tau_{n} y_{j n}$, where

$$
\tau_{n}^{\alpha}=\int_{0}^{+\infty}\left|\int_{\mathbb{R}}(u+n-s)_{+}^{H^{\prime}} \psi(u) d u\right|^{\alpha} d s,
$$

$H^{\prime}=H-1 / \alpha$, and $\left\{y_{j n},|n| \leqslant M 2^{j}, j \geqslant 0\right\}$ is an array of identically distributed symmetric jointly $\alpha$-stable random variables. We first aim to prove

$$
\sup _{n} \tau_{n}<+\infty
$$

To get this uniform bound, we first recall that $\psi$ has its support in $[-D, D]$, so that the integral defining $\tau_{n}^{\alpha}$ can be rewritten as follows:

$$
\tau_{n}^{\alpha}=\int_{0}^{n+D}\left|\int_{-D}^{D}(u+n-s)_{+}^{H^{\prime}} \psi(u) d u\right|^{\alpha} d s .
$$

We cut the domain of integration over $s$ into $[0, n-2 D]$ and $[n-2 D, n+D]$. The first integral is given by

$$
I_{n}^{1}=\int_{0}^{n-2 D}\left|\int_{-D}^{D}(u+n-s)^{H^{\prime}} \psi(u) d u\right|^{\alpha} d s .
$$

We first transform

$$
\int_{-D}^{D}(u+n-s)^{H^{\prime}} \psi(u) d u
$$

through $\ell$ successive integrations by parts. Recalling (9), we see that each time the border terms $I^{m} \psi( \pm D), 0 \leqslant$ $m \leqslant \ell$, vanish. In the end, we obtain 


$$
\begin{aligned}
I_{n}^{1} & =C \int_{0}^{n-2 D}\left|\int_{-D}^{D}(u+n-s)^{H^{\prime}-\ell} I^{\ell} \psi(u) d u\right|^{\alpha} d s \\
& \leqslant C \int_{0}^{n-2 D}(D+n-s)^{\alpha\left(H^{\prime}-\ell\right)} d s \\
& \leqslant C \int_{D}^{+\infty} s^{\alpha(H-\ell)-1} d s,
\end{aligned}
$$

where in the first inequality we used $\ell>H>H^{\prime}$. The integral on the right-hand side is clearly finite and independent of $n$.

The integral over the second domain is given, after a change of variable, by

$$
I_{n}^{2}=\int_{0}^{3 D}\left|\int_{-D}^{D}(u+2 D-s)_{+}^{H^{\prime}} \psi(u) d u\right|^{\alpha} d s \leqslant C \int_{0}^{3 D}\left(\int_{0}^{3 D} u^{H^{\prime}} d u\right)^{\alpha} d s
$$

Recall that $H^{\prime}>0$ for $\alpha<2$ and $H^{\prime}>-1 / 2$ for $\alpha=2$. Therefore, the integral on the right-hand side is again finite and independent of $n$. Putting (19) and (20) together yields (18) as desired.

The following upper bound on $\|R\|$ is a direct consequence of (16) and (18):

$$
\|R\| \leqslant C \sum_{j \geqslant 0} 2^{-(H-\beta-1 / p) j}\left(\sup _{1 \leqslant|n| \leqslant M 2^{j}}\left|y_{j n}\right|\right),
$$

where $\left\{y_{j n},|n|<M 2^{j}, j \geqslant 0\right\}$ is an array of identically distributed symmetric jointly $\alpha$-stable random variables on $\mathbb{R}$.

The end of the proof is now standard and follows [42, Theorem 3.1]. Choose $\delta>0$ such that

$$
\begin{cases}\delta<H-\beta-1 / p & \text { if } \alpha=2 \\ \delta<H-\beta-1 / p-1 / \alpha & \text { if } \alpha<2 .\end{cases}
$$

Let $m$ be a positive integer. Set

$$
d_{j}=2^{(H-\beta-1 / p)(j-m)-\delta|j-m|}
$$

for every $j \geqslant 0$. On the one hand, it is clear from (21) and (22) that

$$
\left\{\left|y_{j n}\right| \leqslant d_{j}, 1 \leqslant|n| \leqslant M 2^{j}, j \geqslant 0\right\} \subset\left\{\|R\| \leqslant C(\delta) 2^{-(H-\beta-1 / p) m}\right\} .
$$

On the other hand, it follows from Lemma 11 (with $h=1$ ) that

$$
\mathbb{P}\left[\left|y_{j n}\right| \leqslant d_{j}, 1 \leqslant|n| \leqslant M 2^{j}, j \geqslant 0\right] \geqslant \exp -C 2^{m} .
$$

Since $H-\beta-1 / p=1 / \gamma$, we obtain

$$
\liminf _{m \rightarrow \infty} 2^{-m} \log \mathbb{P}\left[\|R\| \leqslant C(\delta) 2^{-m / \gamma}\right]>-\infty
$$

which is equivalent to

$$
\liminf _{\varepsilon \downarrow 0} \varepsilon^{\gamma} \log \mathbb{P}[\|R\| \leqslant \varepsilon]>-\infty,
$$

as desired. The proof is complete in the case $p<\infty$. The case $p=\infty$ can be handled exactly in the same way, replacing (13) by

$$
\|R\| \leqslant C \sum_{j \geqslant 0}\left(\sup _{n \in N_{j}}\left|r_{j n}\right|\left\|\psi_{j n}\right\|\right) .
$$


Remarks. (a) It is clear from the above proof that the assumption $\|\cdot\| \in \mathbf{L}(\beta, p)$ is not necessary to get the lower bound. We just need $\|\cdot\| \in \tilde{\mathbf{L}}(\beta, p)$ w.r.t. $\Psi$, a wavelet family generated by parents $\{\varphi, \psi\}$ that are smooth enough. But the required smoothness depends on the parameter $H$, and this would lead to much heavier notations. For this reason we prefer stating Theorem 7 in this form, save for the loss of generality.

(b) There is at least one example where $\tilde{\mathbf{L}}(\beta, p)$ is really more relevant than $\mathbf{L}(\beta, p)$. It is well-known (see [31, Chapter 6]) that $(\eta, p, q)$-Besov semi-norms, $\eta>0$ and $p, q \geqslant 1$, are equivalent to sequential norms on the wavelet coefficients. More precisely,

$$
\left\|\sum_{n} x_{n} \phi_{n}+\sum_{j, n} x_{j n} \psi_{j n}\right\| \sim\left(\sum_{n}\left|x_{n}\right|^{p}\right)^{1 / p}+\left\|\left\{2^{(\eta-1 / p) j}\left(\sum_{n}\left|x_{j n}\right|^{p}\right)^{1 / p}\right\}_{j \geqslant 1}\right\|_{\ell_{q}} .
$$

Hence, with our notations, it is clear that the $(\eta, p, q)$-Besov semi-norm belongs to $\tilde{\mathbf{L}}(\eta-1 / p, p)$ w.r.t. any wavelet basis $\Psi$. Hence, when $p=+\infty$, Theorem 4 yields the existence of the constant for $R$ with $\gamma=(H-\eta)^{-1}$. Note that this rate is in accordance with the results of [42], which covered the range of parameters $\alpha=2$ and $0<H<1$ (with no restriction on $p$ ).

(c) In the Gaussian case, the $\ell$-smooth-finiteness of the semi-norm must hold when $\|R\|$ is a.s. finite. Indeed, if $\mathcal{H}$ denotes the reproducing kernel Hilbert space associated with $R$, then it is well known that $\mathcal{H}$ contains functions with $(H+1 / 2)$ th derivative in $L_{2}$. On the other hand, from a $0-1$ law for Gaussian measures on linear spaces, the finiteness of $\|R\|$ with positive probability yields that $\|f\|<\infty$ for every $f \in \mathcal{H}$. Hence, $\|\cdot\|$ is $\ell$-smooth-finite as soon as $\ell>H+1 / 2$. In the non-Gaussian case, the challenging question whether $\|R\|<\infty$ a.s. implies the $\ell$-smooth-finiteness of the semi-norm remains open.

(d) For the first efficient use of wavelet methods in similar problems to small deviation probability, we refer to [1] where the optimal finite-dimensional approximation of fractional Brownian motion is considered.

\subsection{Proof of Theorem 9}

The outline of the proof is the same as that for Theorem 7, except that we use Schauder system and provide new estimates for the corresponding coefficients. Recall that under our assumptions, $0<H<2$ in the Gaussian case and $1 / \alpha<H<2$ in the non-Gaussian case. Again we set $H^{\prime}=H-1 / \alpha$. We exclude the case $H^{\prime}=0$ where $R$ is Brownian motion: the result follows then directly from Theorem 1 in [41].

Since $R$ is continuous, we can decompose it on $[0,1]$ along the Schauder system (see $[2,41]$ ):

$$
R_{t}=\sum_{j \geqslant 0} \mathcal{R}_{j}(t)+R_{1} t
$$

for every $t \in[0,1]$. Here, for every $j \geqslant 0$,

$$
\mathcal{R}_{j}(t)=\sum_{n=1}^{2^{j}} r_{j n} \psi_{j n}(t)
$$

where we set $t_{j n}^{0}=(n-1) 2^{-j}, t_{j n}^{1}=(n-1 / 2) 2^{-j}, t_{j n}^{2}=n 2^{-j}$ and

$$
r_{j n}=2 R_{t_{j n}^{1}}-R_{t_{j n}^{0}}-R_{t_{j n}^{2}}
$$

We first suppose that $p<\infty$. Condition $(\tilde{\mathrm{G}})$ entails

$$
\left\|\mathcal{R}_{j}\right\| \leqslant C 2^{j \beta}\left(\sum_{n=1}^{2^{j}}\left|r_{j n}\right|^{p}\right)^{1 / p}
$$

Notice that the coefficients $r_{j n}$ can be rewritten as $r_{j n}=\sigma_{j n} y_{j n}$, where 


$$
\sigma_{j n}^{\alpha}=\int_{0}^{+\infty}\left|2\left(t_{j n}^{1}-s\right)_{+}^{H^{\prime}}-\left(t_{j n}^{0}-s\right)_{+}^{H^{\prime}}-\left(t_{j n}^{2}-s\right)_{+}^{H^{\prime}}\right|^{\alpha} d s
$$

(we use the notation $u_{+}=u \wedge 0$ for every $u \in \mathbb{R}$ ), and $\left\{y_{j n}, 1 \leqslant n \leqslant 2^{j}, j \geqslant 0\right\}$ is an array of identically distributed symmetric jointly $\alpha$-stable random variables. For each $1 \leqslant n \leqslant 2^{j}, j \geqslant 0$, we will now give an upper bound on $\sigma_{j n}$ depending only on $j$.

If $n=1$, then

$$
\sigma_{j 1}^{\alpha}=\int_{0}^{2^{-j}}\left|2\left(2^{-(j+1)}-s\right)_{+}^{H^{\prime}}-\left(2^{-j}-s\right)^{H^{\prime}}\right|^{\alpha} d s \leqslant C \int_{0}^{2^{-j}} s^{\alpha H^{\prime}} d s=C 2^{-\alpha H j} .
$$

If $n>1$, then we set $t_{j n}^{-}=(n-2) 2^{-j} \geqslant 0$ and cut the domain of integration in (24) into [0, $\left.t_{j n}^{-}\right]$and $\left[t_{j n}^{-},+\infty\right)$. Reasoning as above, we see that the second integral

$$
I_{j n}^{2}=\int_{t_{j n}^{-}}^{t_{j n}^{2}}\left|2\left(t_{j n}^{1}-s\right)_{+}^{H^{\prime}}-\left(t_{j n}^{0}-s\right)_{+}^{H^{\prime}}-\left(t_{j n}^{2}-s\right)^{H^{\prime}}\right|^{\alpha} d s,
$$

is bounded from above by $C 2^{-\alpha H j}$. We estimate the first integral as follows:

$$
\begin{aligned}
I_{j n}^{1} & =\int_{0}^{t_{j n}^{-}}\left|2\left(t_{j n}^{1}-s\right)^{H^{\prime}}-\left(t_{j n}^{0}-s\right)^{H^{\prime}}-\left(t_{j n}^{2}-s\right)^{H^{\prime}}\right|^{\alpha} d s \\
& =2^{-j} \int_{2}^{n}\left|2\left(t_{j n}^{1}-t_{j n}^{2}+2^{-j} u\right)^{H^{\prime}}-\left(t_{j n}^{0}-t_{j n}^{2}+2^{-j} u\right)^{H^{\prime}}-\left(2^{-j} u\right)^{H^{\prime}}\right|^{\alpha} d u \\
& =2^{-\alpha H j} \int_{2}^{n}\left|2(u-1 / 2)^{H^{\prime}}-(u-1)^{H^{\prime}}-u^{H^{\prime}}\right|^{\alpha} d u \\
& \leqslant C 2^{-\alpha H j} \int_{2}^{\infty} u^{\left(H^{\prime}-2\right) \alpha} d u \\
& =C 2^{-\alpha H j}
\end{aligned}
$$

where the last equality comes from $H<2$. Putting everything together yields now

$$
\sigma_{j n} \leqslant C 2^{-H j}
$$

and, recalling (23),

$$
\left\|\mathcal{R}_{j}\right\| \leqslant C 2^{(\beta+1 / p-H) j}\left(\sup _{1 \leqslant n \leqslant 2^{j}}\left|y_{j n}\right|\right) .
$$

By lower semi-continuity and triangle's inequality, this entails

$$
\|R\| \leqslant C\left(\left|R_{1}\right|+\sum_{j \geqslant 0} 2^{(\beta+1 / p-H) j}\left(\sup _{1 \leqslant|n| \leqslant M 2^{j}}\left|y_{j n}\right|\right)\right)
$$

and since $R_{1}$ has a symmetric $\alpha$-stable law, we can finish the proof as in Theorem 7 . 
Remarks. (a) In the latter proof, the estimate on $I_{j n}^{1}$ becomes too crude when $H \geqslant 2$. This is the principal reason why we need to introduce smoother wavelets in Theorem 7.

(b) As we said before, the proofs of Theorems 7 and 9 work only for continuous processes. The main reason for this comes from Lemma 11. On the one hand, the exclusion of the boundary value $z=\delta+h / \alpha$ in the non-Gaussian case cancels the important case $H=1 / \alpha$. On the other hand, Šidák's inequality handles the $\left\|\mathcal{R}_{j}\right\|$ 's as if they were independent. But if $R$ is discontinuous, then it is possible that its jumps have a significant influence on every level $\mathcal{R}_{j}$, so that our estimate of the series $\sum_{j}\left\|\mathcal{R}_{j}\right\|$ may not be realistic anymore.

(c) In the non-Gaussian case and when $H<\beta+1 / p+1 / \alpha$, it is easy to see that the statements of Theorems 7 and 9 are false, even if $H>\beta+1 / p$. Suppose for example that $H<1 / \alpha$. It is well-known (see [36, Chapter 10]) that a.s.

$$
\sup _{0 \leqslant t \leqslant 1}\left|R_{t}\right|=+\infty
$$

so that Theorems 7 and 9 cannot hold with respect to the supremum norm (i.e. $\mathcal{K}=+\infty$ in Theorem 4 ), although here $\beta=0$ and $p=+\infty$. Similarly, if $H=1 / \alpha$, then clearly

$$
\sup _{0 \leqslant s<t \leqslant 1}\left(\frac{\left|R_{t}-R_{s}\right|}{|t-s|^{\eta}}\right)=+\infty \quad \text { a.s. }
$$

for every $\eta>0$, so that Theorems 7 and 9 cannot hold with respect to any $\eta$-Hölder semi-norm $(\beta=\eta, p=+\infty)$. It is natural to conjecture that these two theorems remain true in full generality when $H=\beta+1 / p+1 / \alpha$, but in view of the above Remark (b), we probably need different methods.

\section{Lower bounds for LMP: finiteness of the constant for continuous LFSM}

Our aim in this section is to extend Theorem 9 to $X$, the continuous LFSM which we defined in Section 2. Recall that $X$ admits an independent decomposition $X=R+M$ where $R$ (resp. $M$ ) is an RLP (resp. an LMP) with the same parameters. The following theorem can be viewed as a generalization of Lemma 3.2 in [23].

Theorem 12. Let $\|\cdot\| \in \tilde{\mathbf{L}}(\beta, p)$ w.r.t. the Schauder system and $M$ be an LMP with parameters $\alpha \in(1,2]$, $H \in(1 / \alpha, 1)$. Suppose that $H>\beta+1 / p$ if $\alpha=2$ and $H>\beta+1 / p+1 / \alpha$ if $\alpha \neq 2$. Then

$$
\lim _{\varepsilon \downarrow 0} \varepsilon^{\gamma} \log \mathbb{P}[\|M\| \leqslant \varepsilon]=0,
$$

where $\gamma=(H-\beta-1 / p)^{-1}$.

Using Theorems 4 and 9 along with the elementary independence argument developed in [23, p. 1334], Theorem 12 yields readily the desired small deviation theorem for $X$ :

Theorem 13. Let $\|\cdot\| \in \tilde{\mathbf{N}}(\beta, p)$ w.r.t. the Schauder system and $X$ be a continuous LFSM with parameters $\alpha \in$ $(1,2], H \in(1 / \alpha, 1)$. Suppose that $H>\beta+1 / p$ if $\alpha=2$ and $H>\beta+1 / p+1 / \alpha$ if $\alpha \neq 2$. Then

$$
\lim _{\varepsilon \downarrow 0} \varepsilon^{\gamma} \log \mathbb{P}[\|X\| \leqslant \varepsilon]=\lim _{\varepsilon \downarrow 0} \varepsilon^{\gamma} \log \mathbb{P}[\|R\| \leqslant \varepsilon]=-\mathcal{K},
$$

where $\mathcal{K} \in(-\infty, 0), \gamma=(H-\beta-1 / p)^{-1}$ and $R$ is the $R L P$ associated to $X$.

We now proceed to the proof of Theorem 12, which is quite analogous to the proof of Theorem 9, except that our estimates of the Schauder coefficients will not be uniform in time-argument since $M$ has a singularity at 0 . 


\subsection{Proof of Theorem 12}

Again we set $H^{\prime}=H-1 / \alpha \in(-1,1)$ and exclude the trivial situation when $H^{\prime}=0$. Since $M$ is continuous, we can decompose it on $[0,1]$ along the Schauder system: for every $t \in[0,1]$

$$
M_{t}=\sum_{j \geqslant 0} \mathcal{M}_{j}(t)+M_{1} t
$$

where

$$
\mathcal{M}_{j}(t)=\sum_{n=1}^{2^{j}} m_{j n} \psi_{j n}(t)
$$

and $m_{j n}=2 M_{t_{j n}^{1}}-M_{t_{j n}^{0}}-M_{t_{j n}^{2}}$ as in the proof of Theorem 9. We first consider the case $p<+\infty$. Condition $(\tilde{\mathrm{G}})$ entails

$$
\left\|\mathcal{M}_{j}\right\| \leqslant C 2^{\beta j}\left(\sum_{n=1}^{2^{j}}\left|m_{j n}\right|^{p}\right)^{1 / p}
$$

and we just need to evaluate the coefficients $m_{j n}=\sigma_{j n} y_{j n}$, where

$$
\begin{aligned}
\sigma_{j n}^{\alpha} & =\int_{0}^{+\infty}\left|2\left(s+t_{j n}^{1}\right)^{H^{\prime}}-\left(s+t_{j n}^{0}\right)^{H^{\prime}}-\left(s+t_{j n}^{2}\right)^{H^{\prime}}\right|^{\alpha} d s \\
& =2^{-H \alpha j} \int_{0}^{+\infty}\left|2(u+n-1 / 2)^{H^{\prime}}-(u+n-1)^{H^{\prime}}-(u+n)^{H^{\prime}}\right|^{\alpha} d u,
\end{aligned}
$$

and $\left\{y_{j n}, 1 \leqslant n \leqslant 2^{j}, j \geqslant 0\right\}$ is an array of identically distributed symmetric $\alpha$-stable random variables. Notice first that from the so-called Cooper's formula

$$
2(u+n-1 / 2)^{H^{\prime}}-(u+n-1)^{H^{\prime}}-(u+n)^{H^{\prime}}=-H^{\prime}\left(H^{\prime}-1\right) \int_{0}^{1 / 2} \int_{u+n-1+\theta}^{u+n-1 / 2+\theta} v^{H^{\prime}-2} d v d \theta .
$$

Clearly, since $H^{\prime}<2$, this entails

$$
\left|2(u+n-1 / 2)^{H^{\prime}}-(u+n-1)^{H^{\prime}}-(u+n)^{H^{\prime}}\right| \leqslant C \min \left\{(u+n-1)^{H^{\prime}-2}, 1\right\} .
$$

We now fix some $h \in(0,1)$ and first estimate $\sigma_{j n}$ when $2^{h j}<n \leqslant 2^{j}$ :

$$
\begin{aligned}
\sigma_{j n}^{\alpha} & \leqslant C 2^{-H \alpha j} \int_{0}^{+\infty}(u+n-1)^{\alpha\left(H^{\prime}-2\right)} d u \\
& \leqslant C 2^{-H \alpha j}(n-1)^{\alpha\left(H^{\prime}-2\right)+1}=C 2^{-H \alpha j} 2^{-h \alpha(2-H) j} .
\end{aligned}
$$

The estimate of $\sigma_{j n}$ when $1 \leqslant n \leqslant 2^{h j}$ is even simpler:

$$
\sigma_{j n}^{\alpha} \leqslant C 2^{-H \alpha j}\left(\int_{1}^{+\infty} u^{\alpha\left(H^{\prime}-2\right)} d u+\int_{0}^{1} C d u\right) \leqslant C 2^{-H \alpha j} .
$$

Recalling (26), we get 


$$
\begin{aligned}
\left\|\mathcal{M}_{j}\right\| & \leqslant C 2^{\beta j}\left(\sum_{n=1}^{2^{h j}} \sigma_{j n}^{p}+\sum_{n=2^{h j}+1}^{2^{j}} \sigma_{j n}^{p}\right)^{1 / p} \sup _{1 \leqslant n \leqslant 2^{j}}\left|y_{j n}\right| \\
& \leqslant C 2^{-(H-\beta) j}\left(2^{h j}+2^{j} 2^{-h(2-H) p j}\right)^{1 / p} \sup _{1 \leqslant n \leqslant 2^{j}}\left|y_{j n}\right| .
\end{aligned}
$$

The balance is attained at $h=(1+(2-H) p)^{-1} \in(0,1)$, whence

$$
\left\|\mathcal{M}_{j}\right\| \leqslant C 2^{-(H-\beta-h / p) j} \sup _{1 \leqslant n \leqslant 2^{j}}\left|y_{j n}\right| .
$$

Using (29) instead of (25), we can proceed as in the proof of Theorem 9 and obtain

$$
\liminf _{\varepsilon \downarrow 0} \varepsilon^{\gamma^{\prime}} \log \mathbb{P}[\|M\| \leqslant \varepsilon]>-\infty,
$$

with $\gamma^{\prime}=(H-\beta-h / p)^{-1}<\gamma$. In particular

$$
\liminf _{\varepsilon \downarrow 0} \varepsilon^{\gamma} \log \mathbb{P}[\|M\| \leqslant \varepsilon]=0,
$$

which completes the proof of Theorem 12 for $p<\infty$.

The case $p=+\infty$ requires more careful estimates. Condition $(\tilde{\mathrm{G}})$ entails

$$
\left\|\mathcal{M}_{j}\right\| \leqslant C 2^{\beta j} \sup _{1 \leqslant n \leqslant 2^{j}}\left|m_{j n}\right| .
$$

Again we fix some $h \in(0,1)$. Using (30), (27), and (28) we can write,

$$
\left\|\mathcal{M}_{j}\right\| \leqslant C 2^{\beta j} \sup _{1 \leqslant n \leqslant 2^{j}} \sigma_{j n}\left|y_{j n}\right| \leqslant C 2^{-(H-\beta) j}\left(\sup _{1 \leqslant n \leqslant 2^{h j}}\left|y_{j n}\right|+2^{-h_{1} j} \sup _{2^{h j}<n \leqslant 2^{j}}\left|y_{j n}\right|\right),
$$

where we set $h_{1}=(2-H) h$. For every integer $m>0$ we focus on the event

$$
\Omega_{m}=\left\{\left|y_{j n}\right| \leqslant d_{j n}(m), 1 \leqslant n \leqslant 2^{j}, j \geqslant 0\right\}
$$

where we set $m_{1}=[m / h], \delta \in(0, H-\beta)$ and

$$
d_{j n}(m)= \begin{cases}2^{(H-\beta)\left(j-m_{1}\right)-\delta\left|j-m_{1}\right|} & \text { if } 1 \leqslant n \leqslant 2^{h j}, \\ 2^{\left(H-\beta+h_{1}\right)(j-m)-\delta|j-m|} & \text { if } 2^{h j}<n \leqslant 2^{j} .\end{cases}
$$

Take now $h_{1}$ small enough such that

$$
H-\beta<H-\beta+h_{1}<(H-\beta) / h .
$$

On the one hand, it is clear that

$$
\Omega_{m} \subset\left\{\sum_{j \geqslant 0}\left\|\mathcal{M}_{j}\right\| \leqslant C 2^{-\left(H-\beta+h_{1}\right) m}\right\} .
$$

On the other hand, it follows from decorrelation argument of Lemma 11 that

$$
\log \mathbb{P}\left[\Omega_{m}\right] \geqslant-C\left(2^{h m_{1}}+2^{m}\right) \geqslant-C 2^{m} .
$$

Using (31) and (32), we can now finish the proof exactly as in the case $p<+\infty$.

Remarks. (a) Contrary to [23], the above proof does not require any entropy argument and relies only on an elementary estimate of the Schauder coefficients.

(b) It would be quite interesting to calculate the optimal rate

$$
\gamma_{0}=\inf \left\{\gamma>0 \mid \lim _{\varepsilon \downarrow 0} \varepsilon^{\gamma} \log \mathbb{P}[\|M\| \leqslant \varepsilon]=0\right\} .
$$

We did not study this question but it seems plausible that $\gamma_{0}=0$ : indeed, $M$ is a $\mathcal{C}^{\infty}$ process on $(0,+\infty)$, and one may expect subexponential rate for its small deviation probabilities. 


\section{Examples: particular semi-norms}

In this section, we place all our results in the context of the previous literature, and show what is new. We try to be as exhaustive as possible as far as RLP's and LFSM's are concerned. We refer to the surveys [26,27] for more information about other processes and for further references.

Everywhere $R$ (resp. $X$ ) will be an RLP (resp. an LFSM) with Hurst parameter $H>0$ and stability index $\alpha \in(0,2]$, whereas $\|\cdot\|$ will be a semi-norm in $\mathbf{N}(\beta, p)$ or $\tilde{\mathbf{N}}(\beta, p)$ for some $\beta \in \mathbb{R}$ and $p \in(0,+\infty]$. We always suppose that $H>\beta+1 / p$ and set $\gamma=(H-\beta-1 / p)^{-1}$ for our small deviation rate. $\mathcal{K}$ will stand for the small deviation constant appearing on the right-hand side of Theorem 4 . Sometimes we will call them just "the rate" and "the constant". Let us begin with the most classical case, which deserves of course a particular mentioning.

\subsection{Brownian motion}

Brownian motion $B$ is an RLP (or an LFSM) with parameters $H=1 / 2$ and $\alpha=2$, hence it clearly satisfies the assumptions of Corollary 10 (or Theorem 13). Notice that here our Theorem 9 just amounts to Stolz's lower bound criterion (Theorem 1 in [41]), so that in the present paper the originality comes only from Theorem 4 . Corollary 10 entails the existence of Brownian small deviation constants under almost all classical semi-norms (with the regrettable exception of certain Besov semi-norms for which we were unable to prove the appropriate superadditivity index). In other words, we get $\lim _{\varepsilon \downarrow 0} \varepsilon^{\gamma} \log \mathbb{P}[\|B\| \leqslant \varepsilon]=-\mathcal{K} \in(-\infty, 0)$, where the dependence of $\gamma$ on the semi-norm $\|\cdot\|$ is given by the following table:

\begin{tabular}{ll}
\hline$\|\cdot\|$ & $\gamma$ \\
\hline Supremum & 2 \\
$L_{p}$ & 2 \\
$\eta$-Hölder & $2 /(1-2 \eta)$ \\
$p$-variation & $2 p /(p-2)$ \\
$(\eta, p)$-Sobolev & $2 /(1-2 \eta)$ \\
$(\eta, \infty, q)$-Besov & $2 /(1-2 \eta)$ \\
\hline
\end{tabular}

The existence of a finite small deviation constant for Brownian motion under $L_{p}$ and supremum norms is a very classical result, and in this situation the constant $\mathcal{K}$ can even be more or less explicitly computed (see $[8,26]$ and the references therein). The existence of the constant under Hölder semi-norms was first proved by Baldi and Roynette [2] - see also [21], and it is actually a direct consequence of Stolz's upper bound criterion (Theorem 2 in [41]) and of the standard subadditivity argument involving the Markov property of Brownian motion. In the three last examples, Stolz's results do provide the right rate, viz.

$$
-\infty<\liminf _{\varepsilon \downarrow 0} \varepsilon^{\gamma} \log \mathbb{P}[\|B\| \leqslant \varepsilon] \leqslant \limsup _{\varepsilon \downarrow 0} \varepsilon^{\gamma} \log \mathbb{P}[\|B\| \leqslant \varepsilon]<0
$$

with the above corresponding $\gamma$, but here the existence of the limit does not follow directly. In these three cases, our result seems to be new.

It is of course a challenging problem to compute these new Brownian constants. As a rule, this kind of (hard) problems requires analytical techniques depending heavily on the choice of the semi-norm, and it seems difficult to find a universal solution.

We stress that the above table matches quite accurately the sample path properties of Brownian motion. It is well-known that $B$ is $\eta$-Hölder if and only if $\eta<1 / 2$ and has finite $p$-variation if and only if $p>2$. Besides, it has finite $(\eta, p)$-Sobolev semi-norm if and only if $\eta<1 / 2$ [14]. 
Besov semi-norms deserve a special remark since no necessary and sufficient conditions seem to be available. Roynette [33] had proved that Brownian motion has finite $(\eta, p, q)$-Besov semi-norm whenever one of the three following situations occurs:

\begin{tabular}{lll}
\hline$\eta$ & $p$ & $q$ \\
\hline$<1 / 2$ & $>1 / \eta$ & $\geqslant 1$ \\
$<1 / 2$ & $=1 / \eta$ & $=1$ \\
$=1 / 2$ & $>2$ & $=+\infty$ \\
\hline
\end{tabular}

Notice that the latter situation is rather specific, since here small deviation probabilities vanish when $\varepsilon$ is small enough, thus the investigation is pointless.

We are able to prove the existence of a finite small deviation constant in the following situation:

\begin{tabular}{lll}
\hline$\eta$ & $p$ & $q$ \\
\hline$<1 / 2$ & $=+\infty$ & $\geqslant 1$ \\
\hline
\end{tabular}

\subsection{Gaussian fractional processes}

In this paragraph $\alpha=2$ and $H>0$, the Brownian case $H=1 / 2$ being implicitly excluded. Up to normalization constant our process $X$ is the usual fractional Brownian motion, while $R$ can be viewed as the fractionally integrated Brownian motion. Before stating our results, we will give a list of local properties of $R$, most of which are classical. As we mentioned in Section 2, all these local properties remain true for $X$ as soon as $H<1$.

$R$ is $\eta$-Hölder (resp. $\eta$-Lipschitz) if and only if $H>\eta$. In particular, $R$ has finite $p$-variation if $H>1 / p$ and only if $H \geqslant 1 / p$ (the boundary value being excluded when $H \geqslant 1 / 2-$ see Theorem 5.4 in [17], but the situation $H=1 / p<1 / 2$ seems to have escaped investigation). Finally, $R$ has finite $(\eta, p)$-Sobolev semi-norm if and only if $H>\eta[14]$.

Less is known about Besov semi-norms. In [13], Proposition 4.1 and Theorem 4.3, it is proved that if $H<1$ and $0<1 / p<\eta$, then $X$ has finite $(\eta, p, \infty)$-Besov semi-norm if and only if $H \geqslant \eta$ (when $H=\eta$, the small deviation probabilities vanish for small $\varepsilon$ ).

With the same notations as in the preceding paragraph, the following table is a direct consequence of Corollary 8 and of Remark (b) at the end of Section 4.3. Recall that it is available for $R$ and for $X$ with the same finite constant $\mathcal{K}$, as soon as $H>\beta+1 / p$.

\begin{tabular}{ll}
\hline$\|\cdot\|$ & $\gamma$ \\
\hline Supremum & $1 / H$ \\
$L_{p}$ & $1 / H$ \\
$\eta$-Hölder & $1 /(H-\eta)$ \\
$p$-variation & $p /(H p-1)$ \\
$(\eta, \infty, q)$-Besov & $1 /(H-\eta)$ \\
\hline
\end{tabular}

If in addition $H<2$, then we can add another line:

\begin{tabular}{ll}
\hline$\|\cdot\|$ & $\gamma$ \\
\hline$(\eta, p)$-Sobolev & $1 /(H-\eta)$ \\
\hline
\end{tabular}


The existence of $\mathcal{K}$ for $X$ under the supremum norm was established in [23], [37] and [38]. In [23], it is also proved for $R$ when $H<1$. When $H \geqslant 1$, the existence of $\mathcal{K}$ is mentioned in [24]. Under $L_{p}$-norms, the right rate for $R$ can be found in [3]. Specialists seem to be aware of the existence of the constant [29], but up to our knowledge this is not explicitly proved in literature. Notice that the case $p=2$ is rather special because of its Hilbert space background. Quite recently, the exact value of $\mathcal{K}$ under the $L_{2}$-norm was found for integrated Wiener process [11,19], and for fractional Brownian motion [9]. Finally, we refer to [22] for non sharp small ball estimates under the Hölder norm for a large class of stationary Gaussian processes, and to [25] for sharp small ball estimates under Sobolev type norms for certain Gaussian processes including $\mathrm{fBm}$. Our results recover everything that is done for gaussian RLP and fBM, sometimes in a sharper form, and provide new estimates in the Besov case.

\subsection{Non-Gaussian stable Lévy processes}

Non-Gaussian symmetric $\alpha$-stable Lévy processes are RLP's with parameters $\alpha<2$ and $H=1 / \alpha$. They have a.s. discontinuous sample paths, hence in the present paper they are only concerned by Theorem 4 .

Recall that they have finite $p$-variation if and only if $p>\alpha$ [7]. In the case $1<\alpha<2$, it is shown in [13] Theorem 6.1 that they have finite $(1 / \alpha, p, \infty)$-Besov norm if and only if $1 \leqslant p<\alpha$.

Our Theorem 4 yields the following table which can be read as in the preceding paragraph, except that here we know nothing a priori about the finiteness of $\mathcal{K}$.

\begin{tabular}{ll}
\hline$\|\cdot\|$ & $\gamma$ \\
\hline Supremum & $\alpha$ \\
$L_{p}$ & $\alpha$ \\
$p$-variation & $\alpha p /(p-\alpha)$ \\
\hline
\end{tabular}

Under the supremum norm, the finiteness of $\mathcal{K}$ dates back to Taylor [44] and Mogulskii [32]. See also [5] for the computation of $\mathcal{K}$ in the completely asymmetric case, and [10] for applications to functional laws of the iterated logarithm. Notice that in this case the existence of $\mathcal{K}$ follows readily from the Markov property, so that our Theorem 4 is a bit useless here. Under $L_{p}$-norms, the existence and the finiteness of $\mathcal{K}$ seems to belong to some ancient folklore, but we could not find any precise reference in the literature. We remark that this follows readily from Theorem 4 and Taylor-Mogulskii's lower bound. Z. Shi also indicated us that in this case the problem of $L_{p^{-}}$ small deviations can be reduced to some specific large deviation probabilities for occupation measures which were previously studied by Donsker and Varadhan. It is shown in [39] that the case $p=2$ is again rather specific since it can be derived from the $L_{p}$-small deviations of Wiener process, thanks to an identity in law due to Donati-Martin, Song, and Yor [16]. Finally, we refer to [40] for the finiteness of $\mathcal{K}$ under the $p$-variation norm.

\subsection{Non-Gaussian fractional processes}

In this paragraph $\alpha<2$ and $H>0$, the case $H=1 / \alpha$ being implicitly excluded. As in the Gaussian framework, we begin with listing local properties of $R$, most of which can be found in the monograph [36]. Again, all these local properties remain true for $X$ when it is well-defined.

$R$ is sample-bounded iff $H \geqslant 1 / \alpha$ and belongs locally to $L_{p}$ if and only if $H>1 / \alpha-1 / p . R$ is $\eta$-Hölder (resp. $\eta$-Lipschitz) if and only if $H \geqslant \eta+1 / \alpha$. This latter fact is less classical and was proved for $X$ by Takashima [43] (see also [20]). Notice that the "if" part is quite straightforward from

$$
\left|R_{t}-R_{S}\right| \leqslant C\|\mathbb{Z}\|_{\infty}|t-s|^{\min \{1, H-1 / \alpha\}}, \quad H \geqslant 1 / \alpha
$$

for all $s, t \in[0,1]$. It is interesting to note that contrary to the Gaussian case, the boundary value $\eta=H-1 / \alpha$ is included here. In particular, this entails that $R$ has finite $p$-variation if and only if $H \geqslant 1 / p+1 / \alpha$ [12]. 
Nothing seems to be known about Sobolev or Besov semi-norms. From Hölder continuity, we see that $R$ has finite $(\eta, p)$-Sobolev semi-norm if $H>\eta+1 / \alpha$, yet this does not seem to be a sharp estimate.

When $R$ is continuous and when $H>\beta+1 / p+1 / \alpha$, we get exactly the same tables as in Section 6.2.

The only result which we are aware of in this direction is due to G. Samorodnitsky [35], who had given just a bit less precise bounds in the small deviation problem for LFSM's $(1 / \alpha<H<1)$ under the supremum norm. In the present paper we find the right rate, prove that the constant exists, and our condition on $H$ is the best possible since $R$ is not bounded anymore when $H<1 / \alpha$. In general, our results match quite accurately the local sample-path properties of $R$. Namely, except for Sobolev and Besov norms, the only situations which are not covered are the critical values of $H$ w.r.t. Hölder and $p$-variation semi-norms.

When $R$ is not continuous, i.e. when $H<1 / \alpha$, the only relevant classical semi-norms are the $L_{p}$-norms, as soon as $H>1 / \alpha-1 / p$. Our Theorem 4 yields $\gamma=1 / H$ but nothing is known about the finiteness of the constant $\mathcal{K}$. We believe that $1 / H$ is still the right rate in this situation.

\section{Concluding remarks}

(a) In general, it is not true that $H$ is the only parameter of a self-similar stable process which is involved in the exponential behavior of its small deviation probabilities. Indeed, [35] delivers an example of an $\alpha$-stable, $H$-sssi process whose small deviation rate depends both on $H$ and $\alpha$. Let us also recall the general results of [34] about stable measures, which entail that

$$
\gamma \leqslant \frac{\alpha}{1-\alpha}
$$

for arbitrary $\alpha$-stable processes with $0<\alpha<1$.

(b) The symmetry assumption on the stable processes is a crucial one, since our proofs are heavily based on two Gaussian results: Anderson's and Šidák's inequalities. Nevertheless, it seems likely that everything remains true as soon as $Z$ is not a subordinator.

(c) Theorem 4 can be easily extended to multi-dimensional RLP's having some symmetry, since they also have the crucial extrapolation-homogeneity property. However, modifying the proof of Theorem 7 in a multidimensional context raises technical difficulties related to Šidák's inequality.

(d) As we indicated before, there are many different generalizations of fractional Brownian motion in nonGaussian case. If $\tilde{X}$ is the well-balanced linear fractional stable motion defined in (3), then the proof of Theorem 4 fails in the absence of extrapolation-homogeneity. However, we believe that the rates should be the same.

(e) Although our results are designed for nice translation-invariant semi-norms, it is worthwhile to mention that there exist elaborated techniques concerning norms that are not translation-invariant. See especially $[4,28,29]$ and [39], where weighted $L_{p}$-norms are handled respectively for Brownian motion, fractional Brownian motion, and symmetric $\alpha$-stable processes.

\section{Acknowledgements}

We are much indebted to Charles Suquet for valuable advice concerning wavelet techniques, and to Werner Linde and Zhan Shi for helpful discussions. We are also grateful to an anonymous referee for a very careful reading of our manuscript, and for making many useful suggestions. This work was carried out during a visit at the Technische Universität Berlin of the second-named author, who would like to thank Michael Scheutzow for his kind hospitality. 


\section{References}

[1] A. Ayache, M. Taqqu, Rate optimality of wavelet series approximations of fractional Brownian motion, J. Fourier Anal. Appl. 9 (5) (2003) 451-471.

[2] P. Baldi, B. Roynette, Some exact equivalents for the Brownian motion in Hölder semi-norm, Probab. Theory Related Fields 93 (4) (1992) 457-484.

[3] E. Belinsky, W. Linde, Small ball probabilities of fractional Brownian sheets via fractional integration operators, J. Theoret. Probab. 15 (3) (2002) 589-612.

[4] P. Berthet, Z. Shi, Small ball estimates for Brownian motion under a weighted sup-norm, Studia Sci. Math. Hung. 36 (1-2) (2001) $275-289$.

[5] J. Bertoin, On the first exit time of a completely asymmetric stable process from a finite interval, Bull. London Math. Soc. 28 (5) (1996) $514-520$.

[6] N.H. Bingham, C.M. Goldie, J.L. Teugels, Regular Variation, Cambridge University Press, Cambridge, 1987.

[7] R.M. Blumenthal, R.K. Getoor, Some theorems on stable processes, Trans. Amer. Math. Soc. 95 (1960) $263-273$.

[8] A.A. Borovkov, A.A. Mogulskii, On probabilities of small deviations for stochastic processes, Siberian Adv. Math. 1 (1) (1991) $39-63$.

[9] J.C. Bronski, Small ball constants and tight eigenvalue asymptotics for fractional Brownian motions, J. Theoret. Probab. 16 (1) (2003) $87-100$.

[10] X. Chen, J. Kuelbs, W.V. Li, A functional LIL for symmetric stable processes, Ann. Probab. 28 (1) (2000) $258-277$.

[11] X. Chen, Quadratic functionals and small ball probabilities for the $m$-fold integrated Brownian motion, Ann. Probab. 31 (2) (2003) 10521077.

[12] V.V. Chistyakov, O.E. Galkin, On maps of bounded $p$-variation with $p>1$, Positivity 2 (1) (1998) 19-45.

[13] Z. Ciesielski, G. Kerkyacharian, B. Roynette, Quelques espaces fonctionnels associés à des processus gaussiens, Studia Math. 107 (2) (1993) 171-204.

[14] M. Csörgö, E. Horváth, Q.-M. Shao, Convergence of integrals of uniform empirical and quantile processes, Stochastic Process. Appl. 45 (2) (1993) 283-294.

[15] I. Daubechies, Ten Lectures on Wavelets, SIAM, 1992.

[16] C. Donati-Martin, S. Song, M. Yor, Symmetric stable processes, Fubini's theorem, and some extensions of the Ciesielski-Taylor identities in law, Stochastic Stochastic Rep. 50 (1-2) (1994) 1-33.

[17] R.M. Dudley, R. Norvaiša, An introduction to $p$-variation and Young integrals. With emphasis on sample functions of stochastic processes, MaPhySto Lect. Notes, vol. 1, 1998, Univ. of Aarhus.

[18] P. Embrechts, M. Maejima, Self-similar Processes, Princeton University Press, Princeton, 2002.

[19] D. Khoshnevisan, Z. Shi, Chung's law for integrated Brownian motion, Trans. Amer. Math. Soc. 350 (10) (1998) $4253-4264$.

[20] N. Kôno, M. Maejima, Hölder continuity of sample paths of some self-similar stable processes, Tokyo J. Math. 14 (1) (1991) $93-100$.

[21] J. Kuelbs, W.V. Li, Small ball problems for Brownian motion and for the Brownian sheet, J. Theoret. Probab. 6 (3) (1993) $547-577$.

[22] J. Kuelbs, W.V. Li, Q.-M. Shao, Small ball probabilities for Gaussian processes with stationary increments under Hölder norms, J. Theoret. Probab. 8 (2) (1995) 361-386.

[23] W.V. Li, W. Linde, Existence of small ball constants for fractional Brownian motions, C. R. Acad. Sci. Paris 326 (11) (1998) $1329-1334$.

[24] W.V. Li, W. Linde, Approximation, metric entropy and small ball estimates for Gaussian measures, Ann. Probab. 27 (3) (1999) $1556-1578$.

[25] W.V. Li, Q.-M. Shao, Small ball estimates for Gaussian processes under Sobolev type norms, J. Theoret. Probab. 12 (3) (1999) 699-720.

[26] W.V. Li, Q.-M. Shao, Gaussian processes: inequalities, small ball probabilities and applications, in: Stochastic Processes: Theory and Methods, in: Handbook of Statistics, vol. 19, 2001, pp. 533-597.

[27] M.A. Lifshits, Asymptotic behavior of small ball probabilities, in: Probab. Theory and Math. Statist., Proc. VII International Vilnius Conference (1998), VSP/TEV, Vilnius, 1999, pp. 453-468.

[28] M.A. Lifshits, W. Linde, Approximation and entropy numbers of Volterra operators with application to Brownian motion, Mem. Amer. Math. Soc. 745 (2002).

[29] M.A. Lifshits, W. Linde, Small deviations of weighted fractional processes and average non-linear approximation, Trans. Amer. Math. Soc., 2002, in press.

[30] D. Marinucci, P.M. Robinson, Alternative forms of fractional Brownian motion, J. Stat. Plann. Inference 80 (1-2) (1999) 111-122.

[31] Y. Meyer, Wavelets and Operators, Cambridge Studies in Advanced Mathematics, Cambridge, 1992.

[32] A.A. Mogulskii, Small deviations in a space of trajectories, Theor. Probab. Appl. 19 (1974) 726-736.

[33] B. Roynette, Mouvement brownien et espaces de Besov, Stochastic Stochastic Rep. 43 (3-4) (1993) 221-260.

[34] M. Ryznar, Asymptotic behavior of stable seminorms near the origin, Ann. Probab. 14 (1) (1986) 287-298.

[35] G. Samorodnitsky, Lower tails of self-similar stable processes, Bernoulli 4 (1) (1998) 127-142.

[36] G. Samorodnitsky, M.S. Taqqu, Stable Non-Gaussian Random Processes, Chapman \& Hall, New York, 1994.

[37] Q.-M. Shao, A note on small ball probability of a Gaussian process with stationary increments, J. Theoret. Probab. 6 (3) (1993) 595-602.

[38] Q.-M. Shao, A Gaussian correlation inequality and its application to the existence of small ball constant, Stochastic Process. Appl. 107 (2) (2003) 269-287.

[39] Z. Shi, Lower tails of quadratic functionals of symmetric stable processes, Prépublication de l'Université Paris-VI, 1999. 
[40] T. Simon, Small ball estimates in $p$-variations for stable processes, J. Theoret. Probab., 2003, in press.

[41] W. Stolz, Une méthode élémentaire pour l'évaluation des petites boules browniennes, C. R. Acad. Sci. Paris 316 (11) (1993) $1217-1220$.

[42] W. Stolz, Small ball probabilities for Gaussian processes under non-uniform norms, J. Theoret. Probab. 9 (3) (1996) 613-630.

[43] K. Takashima, Sample path properties of ergodic self-similar processes, Osaka J. Math. 26 (1) (1989) 159-189.

[44] S.J. Taylor, Sample path properties of a transient stable process, J. Math. Mech. 16 (1967) 1229-1246. 